University of Louisville

ThinkIR: The University of Louisville's Institutional Repository

Electronic Theses and Dissertations

4-2012

\title{
The VEGF quadruplex-forming sequence inhibits lung cancer cell growth.
}

Edward David Muench 1987-

University of Louisville

Follow this and additional works at: https://ir.library.louisville.edu/etd

\section{Recommended Citation}

Muench, Edward David 1987-, "The VEGF quadruplex-forming sequence inhibits lung cancer cell growth." (2012). Electronic Theses and Dissertations. Paper 1022.

https://doi.org/10.18297/etd/1022

This Master's Thesis is brought to you for free and open access by ThinkIR: The University of Louisville's Institutional Repository. It has been accepted for inclusion in Electronic Theses and Dissertations by an authorized administrator of ThinkIR: The University of Louisville's Institutional Repository. This title appears here courtesy of the author, who has retained all other copyrights. For more information, please contact thinkir@louisville.edu. 
THE VEGF QUADRUPLEX-FORMING SEQUENCE

INHIBITS LUNG CANCER CELL GROWTH

\author{
By \\ Edward David Muench \\ B.S., University of Louisville, 2010
}

\begin{abstract}
A Thesis
Submitted to the Faculty of the

University of Louisville

J. B. Speed School of Engineering as Partial Fulfillment of the Requirements for the Professional Degree
\end{abstract}

MASTER OF ENGINEERING

Department of Bioengineering

April 2012 

THE VEGF QUADRUPLEX-FORMING SEQUENCE INHIBITS LUNG CANCER CELL GROWTH

Submitted by:

Edward David Muench

A Thesis Approved On

(Date)

by the Following Reading and Examination Committee:

Donald M. Miller, M.D., Ph.D., Thesis Director

Kara J. Sedoris, Ph.D.

Andre M. Gobin, Ph.D.

Hermann B. Frieboes, Ph.D.

Balaji Panchapakesan, Ph.D. 


\section{ACKNOWLEDGEMENTS}

First off, I would like to thank Dr. Donald Miller for taking me on as a co-op student and introducing me to the vitally important and fascinating world of cancer research. He not only helped me discover my dream job, but he has also set an outstanding example of how to be an extraordinary physician scientist that I one day hope to take after. I also want to thank all of the members of the Miller/Bates lab who have helped me learn how fun life can be as a scientist and have inspired me to keep improving no matter what. Thank you to Sheila Thomas for her help with the stability experiments, and to Dr. Tariq Malik for his help with the in vivo imaging studies. Special thanks to Dr. Kara Sedoris, who had the patience and fortitude to work with me every day. I enjoyed every minute of it, and couldn't have done it without her!

I also want to thank Dr. Frieboes and Dr. Panchapakesan for being part of my committee. I appreciate all of your hard work and advice which has been extremely helpful. I also want to thank Dr. Andre Gobin for being such a great professor and for sponsoring my independent study, which was a very enjoyable experience!

Thank you to my loving parents, Ed and Carol Muench, who have supported and encouraged me to do my best at everything I do. Lastly and most importantly, I would like to thank my fiancée, Tricia Pangallo, for the constant moral support that she has provided as well as for continuing to inspire me every single day. 


\begin{abstract}
Vascular endothelial growth factor (VEGF), a commonly overexpressed oncogene in a variety of malignancies including non-small cell lung cancer (NSCLC), is a key regulator of angiogenesis promoting tumor survival, growth, and metastasis. The promoters of several cancer-related genes, including VEGF, contain disproportionate sequences within nuclease hypersensitivity regions capable of forming quadruplex (fourstranded) DNA. The specific quadruplex forming sequence of interest is the 20 base pair polyG/polyC tract that codes for the VEGF promoter (VEGFq), which is located in the proximal promoter region upstream of the transcription initiation site. This oligonucleotide has been shown to have significant growth hindering effects when introduced in the NSCLC cell line, A549.

To determine the biological role of VEGFq on non-small cell lung cancer in vitro, cells were treated with either VEGFq or the corresponding mutant sequence (MutVEGF), which lacks the runs of guanines necessary for quadruplex formation. Circular dichroism spectroscopy confirmed that VEGFq formed a parallel quadruplex, while the MutVEGF sequence did not form a quadruplex structure. It is hypothesized that the considerable growth inhibition is caused by hindering the VEGF signaling pathway. The VEGF signaling cascade, which is normally triggered by VEGF binding to FLK1 (VEGF Receptor 2), is unable to function adequately due to decreased VEGF protein levels. This causes a disruption in the phosphorylation of target proteins including ERK1/2 and $\mathrm{AKT} / \mathrm{PKB}$, which in turn decreases overall cell proliferation. This observed decline in overall cell proliferation corresponded to decreased levels of VEGF protein expression,
\end{abstract}


indicating that there is a direct correlation between the treatment and the changes in proliferation. A single treatment of A549 NSCLC cells with VEGFq caused a significant dose and time-dependent decrease in cell viability after 3 and 6 days as determined by MTT assay. Parallel treatment of nontransformed human fibroblast cells with VEGFq showed no changes in growth, demonstrating the cancer specificity of VEGFq. Cell cycle analysis showed no changes in cell phases at 24, 48, 72 and 96 hours, indicating that VEGFq's effects due to a mechanism other than cell cycle arrest. Confocal microscopy and flow cytometry after 72 hours showed significant uptake and nuclear localization of VEGFq, but not MutVEGF. Boyden chamber invasion/migration measurement shows that the VEGFq treated cells have decreased cell movement, indicative of possible antiangiogenic effects as well.

To determine the biological role of VEGFq on non-small cell lung cancer in vivo, A549 cells were injected into nude mice and grown for 10 days prior to daily IP injections of $10 \mathrm{mg} / \mathrm{kg}$ VEGFq or the control vehicle for 14 days. Tumor progression was physically measured using calipers three times a week. Fluorescent imaging was used to detect the presence, stability, and distribution of Alexa Fluor 750-labeled VEGFq after injection into the mouse to ensure localization in the targeted tumor.

These results demonstrate that A549 cells treated with VEGF quadruplex-forming oligonucleotides experience a dramatic decrease in cell proliferation, suggesting that VEGFq may have significant therapeutic implications for the treatment of non-small cell lung cancer. 


\section{TABLE OF CONTENTS}

$\underline{\text { Page }}$

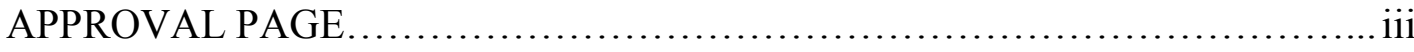

ACKNOWLEDGMENTS ............................................... iv

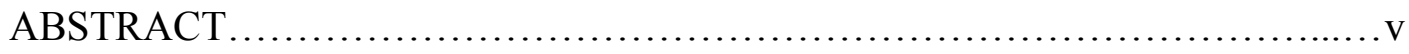

NOMENCLATURE......................................................... ix

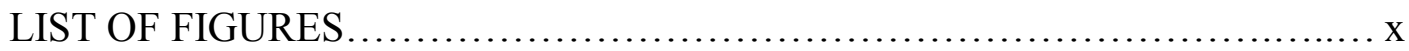

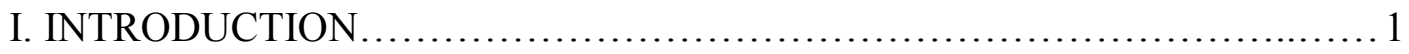

1.1 Lung Cancer and Current Treatments............................... 1

1.2 Vascular Endothelial Growth Factor and its Receptors.................. 2

1.3 VEGF Signaling and Cancer...................................... 4

1.4 Oligonucleotide Therapy..................................... 5

1.5 G-Quadruplex DNA for Cancer Therapy.......................... 7

1.6 Preliminary Data................................................ 8

1.7 Purpose of the Study.......................................... 10

1.8 Hypotheses........................................................ 10

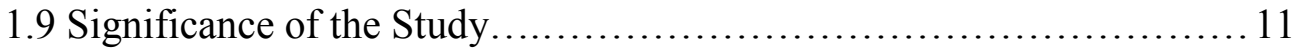

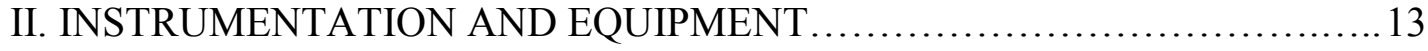

2.1 Circular Dichroism Spectrometer.................................. 13

2.2 Spectrophotometer......................................... 13

2.3 Flow Cytometer.............................................. 13

2.4 Confocal Microscope.............................................. 14

2.5 In Vivo Photon Imager............................................ 14

III. PROCEDURE....................................................... 15

3.1 General....................................................... 15

3.2 Circular Dichroism Spectroscopy.................................. 16

3.3 MTT Assay.................................................... 17

3.4 Analysis of VEGFq and MutVEGF Uptake....................... 17

3.5 Cell Cycle Analysis..............................................19

3.6 Serum and Intracellular Stability of VEGFq....................... 20

3.7 Western Blot analysis......................................... 20

3.8 p-FLK-1 ELISA............................................... 21

3.9 Boyden Chamber Invasion/Migration Assay.........................22

3.10 VEGFq In Vivo Activity....................................... 23

3.11 VEGFq In Vivo Stability and Biodistribution................................24

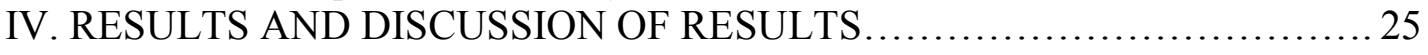

4.1 VEGFq forms a parallel quadruplex in solution.................... 25 
4.2 VEGFq inhibits NSCLC cell proliferation.......................... 26

4.3 Enhanced cellular uptake of VEGFq.............................. 27

4.4 VEGFq and MutVEGF do not cause a change in cell cycle............29

4.5 Enhanced VEFGq serum and cytoplasmic stability...................30

4.6 VEGFq treatment decreases VEGF protein levels......................30

4.7 VEGFq alters activation of FLK-1 and downstream signaling......... 31

4.8 VEGFq inhibits NSCLC cell invasion.............................. 32

4.9 VEGFq inhibits in vivo NSCLC cell proliferation........................33

4.10 Enhanced VEGFq in vivo stability and tumor localization.............34

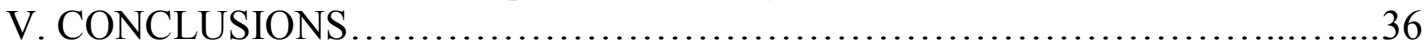

VI. RECOMMENDATIONS ............................................. 37

\section{APPENDIX I}

REFERENCES........................................................... 43

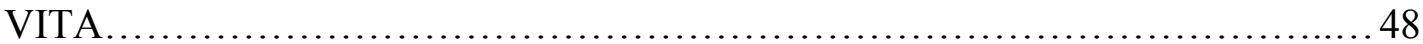




\title{
NOMENCLATURE
}

\author{
BSA $=$ Bovine Serum Albumin \\ $\mathrm{CD}=$ Circular Dichroism Spectroscopy \\ ChIP $=$ Chromatin Immunoprecipitation \\ FBS $=$ Fetal Bovine Serum \\ FLK-1 = VEGF Receptor 2 \\ HRP $=$ Horseradish Peroxidase \\ $\mathrm{IP}=$ Intraperitoneal \\ MTT $=$ 3-(4,5-dimethylthiazol-2yl)-2,5-diphenyltetrazolium bromide \\ MutVEGF $=$ Mutant VEGF Sequence \\ NSCLC $=$ Non-Small Cell Lung Cancer \\ ODN = Oligodeoxynucleotide \\ PBS = Phosphate Buffered Saline \\ ROI $=$ Region of Interest \\ S100 $=$ Cytoplasmic Extract \\ VEGF $=$ Vascular Endothelial Growth Factor \\ VEGFq $=$ Quadruplex-Forming VEGF Promoter Sequence
}




\section{LIST OF FIGURES}

PRELIMINARY FIGURE 1 - Quantitation of the basal protein levels of commonly overexpressed oncogenes whose promoters are guanine rich and the changes in growth due to treatment with each respective oncogene's G-Quadruplex ODN in A549 NSCLC.

FIGURE 1 - Representative CD scan of the quadruplex forming oligonucleotide VEGFq and the non-quadruplex forming oligonucleotide MutVEGF .25

FIGURE 2 - MTT dose response time courses for VEGFq and MutVEGF in A549, H1299, and Hs27 cells.

FIGURE 3 - Cellular uptake and localization of VEGFq and MutVEGF in A549 and Hs27 cells

FIGURE 4 - Cell cycle analysis of A549 cells treated with VEGFq or MutVEGF.

FIGURE 5 - Comparison of VEGFq and MutVEGF stability in serum and in cell extract.

FIGURE 6 - Western Blot results of VEGFq and MutVEGF treated A549 cells 
FIGURE 7 - Schematic of VEGFR-2 signaling and Western Blot and ELISA analysis of changes in receptor and downstream protein and phosphorylation levels due to VEGFq and MutVEGF treatment in A549 cells.....................32

FIGURE 8 - Invasion/Migration analysis of A549 cells treated with VEGFq or MutVEGF

FIGURE 9 - In Vivo change in tumor growth of xenografted A549 cells treated with VEGFq or MutVEGF................................................. 34

FIGURE 10 - In Vivo Alexa Fluor 750 labeled VEGFq fluorescent intensity in xenografted A549 tumors................................................ 35 


\section{INTRODUCTION}

\subsection{Lung Cancer and Current Treatments}

Lung cancer is a highly prevalent disease with an estimated 250,000 new diagnoses in 2011 in the US alone and is the leading cause of cancer deaths in the US, with a staggering 5 -year survival rate of only $15.7 \%$. Lung cancer can be broken down into two subtypes known as small cell lung cancer and non-small cell lung cancer (NSCLC) depending on the tumor morphology (NCI, 2011). NSCLC comprises $80.4 \%$ of lung cancers and can be further classified as adenocarcinoma, squamous cell carcinoma, or large cell carcinoma (NCBI, 2011, Travis, 1995). Adenocarcinomas are the most common form of lung cancer, accounting for $44 \%$ of NSCLC, and are typically found in the outer regions of the lung. Another $37.5 \%$ of NSCLC are squamous cell carcinomas, which are usually found in the center of the lung next to a bronchus. The less common large cell carcinomas account for the other $18.5 \%$, and can be found in any part of the lung. Smoking has been identified as the primary cause of most cases of adenocarcinoma and squamous cell carcinoma; however, it is a common misconception that smoking is the only way to develop lung cancer. In fact, some people who have never smoked have gotten lung cancer (NCBI, 2011, Bryant, 2007). Although it is often difficult to pinpoint what causes each patient's lung cancer, some common risk factors for NSCLC include long term exposure to high levels of: air or drinking water pollution, asbestos, coal products, mustard gas, preservatives, and formaldehyde (NCBI, 2011). Despite lung cancer research funding of over 281 million dollars in 2010 alone, not much progress has been made in the way of improving patient survival rates (NCI, 2011). 
Conventional treatment options for non-small cell lung cancer (NSCLC) involve a long list of regimens, including 1) cytotoxic combination chemotherapy with the platinum based drugs cisplatin or carboplatin as well as paclitaxel, gemcitabine, docetaxel, vinorelbine, irinotecan, and pemetrexed; or, 2) bevacizumab and cetuxemab; or 3) epidermal growth factor receptor tyrosine kinase inhibitors; or, 4) cisplatin, vinorelbine, and cetuximab; or, 5) maintenance premetrexed; or, 6) external beam radiation therapy; or, 7) endobronchial laser therapy and/or brachytherapy. The specific treatment route selected for patients depends on several factors and disease specifics including but not limited to: tumor histology, patient age versus comorbidity, performance status, presence, number, and location of metastases, EGFR expression and/or mutation, and previous treatments received (NCI, 2011). All of these therapies are accompanied by a number of side effects ranging from mild to severe that are manifested as a result in damage to patients' normal cells due to their non-specificity for cancer. The lethal nature of NSCLC demands that a more effective and selective therapy be developed to improve outcomes and reduce the burden of unwanted side effects.

\subsection{Vascular Endothelial Growth Factor and Its Receptors}

The Vascular Endothelial Growth Factors (VEGFs) are a family of five secreted dimeric proteins capable of acting upon many different cell types, but are mainly associated with endothelial cell stimulation. VEGF members include VEGFA, VEGFB, VEGFC, VEGFD, and placenta growth factor (PLGF) that regulate the growth of new blood vessels in embryos (vasculogenesis), the growth of blood vessels from existing vasculature (angiogenesis), and overall vascular maintenance in adults (Olsson, 2006). 
Prior to the discovery of the other subtypes, VEGF referred to VEGFA, which is the primary member of its growth factor family, and the two terms are typically interchangeable (Loureiro, 2005).

VEGFA is an eight exon long gene located on chromosome 6 that undergoes premRNA splicing to produce proteins from 19 of its 25 unique transcripts; however, only six of these have been highly classified (ENSEMBL, 2011, Robinson, 2001, Hilmi, 2011). These isoforms are annotated as VEGFxxx, where the $\mathrm{xxx}$ represents the number of amino acids present minus the signal peptide. The most common are 121, 165, and 189, which have been found to exist as an "a" or "b" splice variant. VEGFxxxa forms arise from the proximal splicing of exon 8, and VEGFxxxb forms are created from the distal splicing of exon 8. As expected, VEGFxxxa forms have been shown to be proangiogenic. Curiously however, evidence has been found that suggests that VEGFxxxb forms have an inhibitory effect on angiogenesis (Hilmi, 2011).

VEGF is capable of acting on cells through the binding to its tyrosine kinase receptors (VEGFR), numbered one through three. These receptors contain seven varying extracellular immunoglobulin-like domains allowing them to bind different forms of VEGF with differing affinities. Additionally, they contain conserved trans-membrane domains, anchoring them to the plasma membrane, and intracellular tyrosine kinase domains allowing them to participate in signal transduction. VEGFR-1 and VEGFR-2 bind several forms of VEGF, but have the highest affinity for VEGFA. VEGFR-3 does not bind VEGFA, only the VEGFC and VEGFD forms. Despite its capability to bind VEGFA, VEGFR-1 activation has no apparent effects on proliferation, migration, or 
cytoskeletal remodeling can be detected. Conversely, VEGFR-2 activation has been shown to induce these processes (Robinson, 2001).

\subsection{VEGF Signaling and Cancer}

VEGF is often highly overexpressed in lung cancer and is commonly associated with poor prognosis, making it an attractive target for therapeutic agents for treatment of NSCLC (O'Byrne, 2006, Robinson, 2001, Yuan, 2000). In order for VEGF to transmit its message to cancer cells, a series of signaling steps must occur (Figure 7A). Firstly, VEGF must be secreted and bind to VEGFR-2 on the target cell, acting through an autocrine or paracrine mechanism (Mendel, 2000). Receptor binding then stimulates a cascade through several downstream kinases, notably ERK, p38, and Akt. These kinases then trigger the up-regulation of proliferation, angiogenesis, vascular permeability, and cell survival (Kanehisa Laboratories, 2009). These processes have been identified to be critical for tumor survival, growth, and metastasis of lung cancer (Molina, 2006).

Bevacizumab, a monoclonal antibody to VEGF, is a recent development in targeted VEGF therapy that has shown promising results in patient studies; however, patients treated with bevacizumab still experience adverse effects of the drug and even deaths due to pulmonary hemorrhage, gastrointestinal hemorrhage, central nervous system infarction, gastrointestinal perforation, myocardial infarction, or neutropenic sepsis (Cao, 2011, NCI, 2011). VEGF-targeted therapeutics provide an important and necessary weapon against NSCLC to ensure that the tumor does not receive a blood supply adequate enough to survive, thrive, and spread to other regions of the body, yet 
improvement upon current VEGF directed therapeutics is necessary to enhance patient outcomes and reduce treatment related harm.

Another method of inhibiting VEGF-induced tumor growth involves targeting VEGFR-2 so that cannot transmit its signaling cascade correctly. Two small molecule tyrosine kinase inhibitors (TKI) being investigated include sorafenib and sunitinib. These multi-targeted inhibitors do not specifically act upon VEGFR-2; however, they work to inhibit multiple kinases. Although they both show promise in other types of cancer, they do not appear to be any benefit to lung cancer patients (Jain, 2006). In fact, the addition of sorafenib to treatment regimens for squamous cell lung cancer increased patients' risk of mortality (Chustecka, 2008). Another TKI that is specific to VEGFR-2 is known as semaxanib. Despite its advancement to phase III clinical trials in colorectal cancer, it failed to meet the efficacy goals, and as a result the trials were canceled prior to completion. Further drug development, including investigation as a treatment for lung cancer, was not pursued due to these poor results (Jain, 2006). Even though TKI therapies show promise for some cancer types, it is apparent that this current generation of drugs do not show enough efficacy for the treatment of NSCLC.

\subsection{Oligonucleotide Therapy}

One class of gene-specific therapeutic agents that has been examined as a potential cancer therapy is known as an antisense oligonucleotide. First discovered around 1980, these naturally occurring single stranded oligonucleotides are complementary to the RNA sequence of their target. Synthetic antisense drugs have been created to inhibit the expression of a gene through binding to the targeted sequence, 
inhibiting translation of the gene (Zhang, 2005). While this gene-specific approach aims to improve upon whole cell effects by only inhibiting the undesired gene, it fails to exclusively target cancer cells, exhibiting highly toxic side effects in all cells. Some additional barriers against successful antisense therapy include low serum stability, low affinity for target molecules, low cellular uptake, and vulnerability to nucleolytic degradation (Kurreck, 2003). While the concept is promising, the setbacks that accompany antisense therapy make it an unattractive option for the widespread treatment of cancer.

Another gene-specific therapeutic agent that has been developed recently is the Gquadruplex forming oligonucleotide. These oligonucleotides are guanine-rich, and possess four runs of at least three guanines, each separated by between one and seven bases. Through Hoogsteen hydrogen bonding, four nonadjacent guanines can associate with one another, forming a guanine tetrad. Furthermore, these guanine tetrads are capable of stacking upon one another, establishing a G-quadruplex (Gubala, 2004). These structures occur naturally throughout the human genome, most notably in telomeres, which are found at the ends of chromosomes, as well as in the promoter regions of an estimated $43 \%$ of all genes, including VEGF. While telomeric G-quadruplex formation is believed to play a role in the protection of chromosomes, those formed in promoter sequences are thought to negatively regulate transcription of the downstream gene via steric blockage (Huppert, 2008). This suggests that G-quadruplexes could be an attractive agent to selectively inhibit the expression of targeted genes. 


\subsection{G-Quaduplex DNA for Cancer Therapy}

Until recently, the biological significance of G-quadruplex oligonucleotides has remained unknown. Just over a decade ago, AS1411, a G-rich oligonucleotide designed as a control sequence for another oligonucleotide being investigated as an inhibitor of gene expression in cancer, exhibited strange properties during the initial studies. The sequence actually caused far greater growth inhibition of the cancer cells compared to the oligonucleotide being investigated. Focus quickly shifted to determine the reason and mechanism behind the effects caused by this random sequence. During this investigation, it was noted that this G-rich sequence overcomes all of the setbacks of conventional antisense oligonucleotide therapies. Namely, AS1411 is highly resistant to degradation by serum and cellular nucleases, it is taken up by cells without the use of transfection reagents, and most importantly, it does not have toxic effects on non-cancerous cells. Since its initial discovery, AS1411 has been shown to be effective in inhibiting the growth of over 60 different cancer cell lines. Due to its high reactivity in cancer and low toxicity to non-transformed cells, it has progressed through phase II clinical trials, exhibiting promising results in both renal cell carcinoma and acute myeloid leukemia (Bates, 2009).

The success of AS1411 has inspired an abundance of studies aimed at not only solving the structure and mechanisms of G-quadruplex oligonucleotides, but to also seek out other G-rich sequences that can be used as cancer therapies. The promoter regions of many oncogenes are G-rich and are believed to be capable of being specifically targeted and suppressed by their own specific sequence through the formation of a G-quadruplex. One such synthetic G-quadruplex forming oligonucleotide that codes for the promoter 
sequence of the common oncogene c-myc is known as PU27. Treatment of leukemic cells with PU27 has been shown to selectively kill these malignant cells through down regulation of c-Myc, while overcoming the same setbacks of antisense therapy that AS1411 does (Sedoris, 2011). This breakthrough suggests that the targeting of VEGF and other genes containing G-rich promoter sequences (e.g. c-Myc, HIF-1, Bcl-2, etc) with their corresponding oligonucleotides may possess therapeutic efficacy as cancer-specific drugs (Qin, 2008).

\subsection{Preliminary Data}

The work in this section was not performed by the author, but has been included to support the author's hypothesis and rationale for pursuing this study. The preliminary experiments were performed and analyzed by Dr. Kara Sedoris, Sheila Thomas, Cortney Clarkson, Campbell Grant, and Dr. Donald Miller at the James Graham Brown Cancer Center.

In order to identify a molecular target for the treatment of NSCLC with Gquadruplex oligodeoxynucleotides (ODNs), the A549 NSCLC protein expression levels of common oncogenes containing G-rich promoters (HIF-1, VEGF, BCL-2, and c-Myc) were determined by Western Blot analysis (Preliminary Figure 1A). After normalizing each oncoprotein to the housekeeping protein $\beta$-actin, VEGF was identified as having the highest expression out of the four selected proteins, distinguishing it as an attractive target in A549 cells. Furthermore, the four oncogenes' Quadruplex-forming ODNs' effects on cell growth of A549 cells were determined using an MTT assay (Preliminary Figure 1B). This test elucidated that the VEGF ODN drastically inhibited A549 cell 
growth compared to the other ODNs, perhaps due to the cells' higher expression of VEGF. These preliminary findings suggest that the VEGF quadruplex forming ODN could be a potential therapy for NSCLC, warranting further study and development of this ODN.
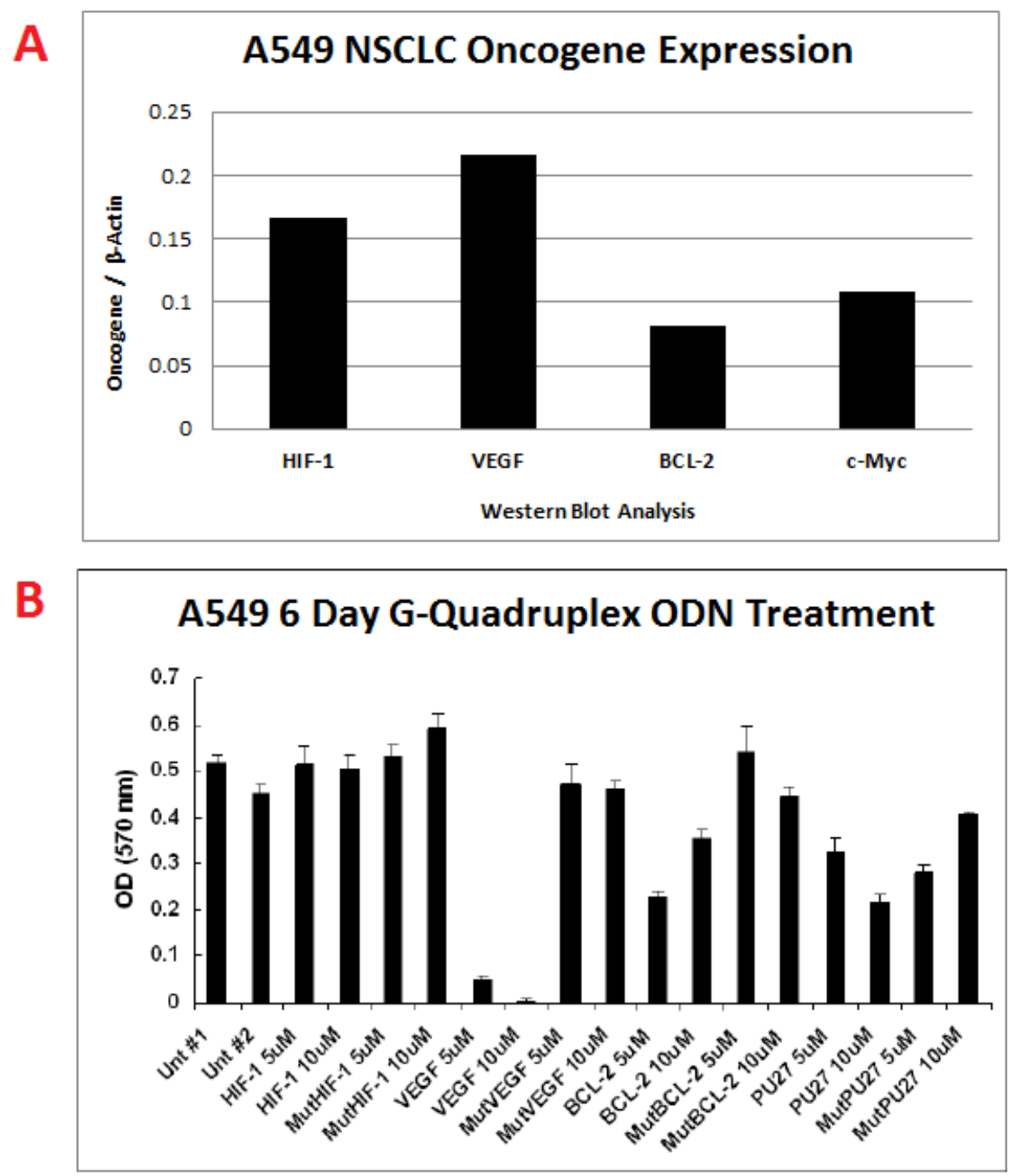

PRELIMINARY FIGURE 1 - Quantitation of the basal protein levels of commonly overexpressed oncogenes whose promoters are guanine rich and the changes in growth due to treatment with each respective oncogene's GQuadruplex ODN in A549 NSCLC. 


\subsection{Purpose of Study}

The purpose of this study was to determine the biological significance of VEGFq, the G-quadruplex forming oligonucleotide that codes for the proximal promoter sequence, as a possible treatment for NSCLC. Characterization of oligonucleotide secondary structure, quantitation of cell proliferation and invasion, quantitation of oligonucleotide uptake, localization, and stability, quantitation of changes in protein expression and phosphorylation, and quantitation of in vivo oligonucleotide activity, distribution, and stability were performed. Additionally, the data collected in this study can be utilized to plan future experiments to further model the uptake and action mechanisms of this novel compound.

\subsection{Hypotheses}

1) In vitro treatment of NSCLC cells with VEGFq inhibits cell growth and invasion by decreasing VEGF expression.

2) The in vitro VEGFq-induced decrease in VEGF expression down regulates FLK-1 activation and signaling through downstream kinases.

3) In vivo treatment of xenografted NSCLC cells in nude mice with VEGFq selectively inhibits NSCLC cell growth.

4) In vivo injection of VEGFq into nude mice xenografted with NSCLC cells primarily localizes and remains in the tumors compared to non-transformed cells. 


\subsection{Significance of the Study}

VEGF is a key protein in a variety of cancers including lung cancer that triggers cells to survive, thrive, and eventually spread to other parts of the body. Inhibition of VEGF signaling is believed to be an important process in halting lung cancer growth and metastasis; however, a suitable drug that can accomplish this without causing significant side effects is yet to be discovered. VEGFq is believed to be capable of filling this void. Additionally, if current studies are successful, VEGFq may be examined for therapeutic efficacy in other cancer types in which VEGF is overexpressed. Through the development and characterization of VEGFq, our group hopes to add another genespecific and cancer-specific agent to the arsenal of next generation oligonucleotides for utilization in the battle against cancer.

Personalized medicine, or the specific selection of treatment options based upon the individual patients' genetic profile rather than the broad treatment of a disease, has been an attractive yet unrealized idea. This is most likely due to the non-targeted and globally acting nature of most drugs as well as the unavailability of the necessary genespecific drugs. Without the ability to specifically target genes, personalized medicine cannot be practiced; however, quadruplex-forming drugs like VEGFq present a possible opportunity for the development of personalized medicine. Not only does VEGF have a quadruplex in its promoter sequence, but the promoters of many other oncogenes have been identified as being capable of forming quadruplexes. Just a few of these include cMyc, c-kit, KRAS, HIF-1, Bcl-2, and RET, with many others yet to be identified and characterized (Qin, 2008). Through the use of quadruplex drugs that specifically target and silence these genes in cancer, the practice of personalized medicine may be possible. 
Individual patients' genomes could be sequenced to identify the key oncogenes activated in their specific cancer, which will allow physicians to tailor their treatment regiments to include only the quadruplex drugs needed. Although further investigation and development of other quadruplex-forming drugs is necessary prior to their widespread use, this class of cancer drugs theoretically satisfies the current deficiencies of personalized medicine. 


\section{INSTRUMENTATION AND EQUIPMENT}

This section contains information regarding the instrumentation and equipment used during the completion of this thesis.

\subsection{Circular Dichroism Spectropolarimeter}

Circular Dichroism Spectra were recorded on a Jasco-810 spectropolarimeter (Jasco, Easton, MD), using a quartz cell of $1 \mathrm{~mm}$ optical path length, an instrument scanning speed of $200 \mathrm{~nm} / \mathrm{min}$, response time of $2 \mathrm{sec}$, and scans were performed over a wavelength range of $340 \mathrm{~nm}$ to $220 \mathrm{~nm}$ at room temperature $(\sim 25 \mathrm{C})$.

\subsection{Spectrophotometer}

Absorbances of 96 well plates were determined by using a BioTek Synergy HT spectrophotometer (BioTek, Winooski, VT). The selected wavelength of light used to read plates varied depending on experimental conditions.

\subsection{Flow Cytometer}

Cell cycle and uptake of FITC-labeled ODNs were determined using a FACS Calibur flow cytometer (BD Biosciences, San Jose, CA). Initial cytometer settings were optimized for each untreated cell type used, and saved for use during subsequent experiments to maximize consistency between replicates. 


\subsection{Confocal Microscope}

Cells were visualized by confocal microscopy with an Olympus Fluoview FV500 (Olympus, Center Valley, PA) laser scanning microscope. A $493 \mathrm{~nm}$ laser was used to visualize the DAPI fluorphore and a $358 \mathrm{~nm}$ laser was used to visualize the FITC fluorophore.

\subsection{In Vivo Photon Imager}

In vivo uptake and biodistribution of Alexa Fluor 750-labeled VEGFq was imaged using a Photo Imager RT photon imager, using an excitation wavelength of $750 \mathrm{~nm}$. Fluorescence integration mode was selected, and a total of 750,000 events were collected over an acquisition time of 4 seconds to quantitate signal intensity. Images were generated by capturing $1000 \mathrm{~ms}$ per frame. 


\section{PROCEDURE}

\section{$\underline{3.1 \text { General }}$}

A549 adenocarcinomic human alveolar basal epithelial cells, H1944 adenocarcinomic non-small cell lung cancer cells, H1299 non-small cell lung cancer carcinoma cells, and Hs27 human fibroblast cells (ATCC) were maintained in DMEM media supplemented with $10 \%$ Fetal Bovine Serum (FBS) and $100 \mathrm{U} / \mathrm{mL}$ penicillin/streptomycin at $5 \% \mathrm{CO}_{2}$ and $37{ }^{\circ} \mathrm{C}$. The three lung cancer cell lines were used to determine the biological significance of the VEGFq oligonucleotide as a potential treatment for NSCLC while the readily available non-cancerous fibroblast cells were used as a control for growth inhibition studies to demonstrate VEGFq's cancer specificity.

Synthesized oligodeoxynucleotides (ODNs) (Oligos Etc.), delivered as a lyophilized powder, were reconstituted in RNAse/DNAse free ultrapure $\mathrm{DH}_{2} \mathrm{O}$ (Invitrogen) to a stock concentration of $500 \mu \mathrm{M}$ upon receipt and stored at $-20{ }^{\circ} \mathrm{C}$ when not in use. Prior to treatment, the oligonucleotides were thawed at room temperature and then boiled at $95^{\circ} \mathrm{C}$ for $5 \mathrm{~min}$. The boiling is hypothesized to increase the activity of the oligonucleotides, which is believed to be due to the denaturing of the secondary structures that formed during cold storage. The VEGFq sequence, which contains runs of three or more adjacent guanines that are required for quadruplex formation, was the main oligonucleotide of interest in this study, while MutVEGF was used as an oligonucleotide control. The general synthesis and preparation methods of both oligonucleotides were identical; however, their primary sequences differ. MutVEGF is essentially the same 
sequence as VEGFq except that several guanines have been mutated to cytosines so that it does not contain the runs of three or more adjacent guanines. Without the clusters of guanines that are necessary for quadruplex formation, it is hypothesized that MutVEGF does not form a quadruplex. It can then be used as a control in all studies to ensure that the effects of VEGFq are not simply due to the introduction of foreign DNA, but are instead sequence specific.

Cells in logarithmic growth phase were plated and treated with $10 \mu \mathrm{M}$ (final concentration unless otherwise specified) of VEGFq or the respective control, MutVEGF, for various time intervals and collected for subsequent biochemical analysis. The ensuing data was recorded and analyzed using Microsoft Excel, unless otherwise noted.

\section{$\underline{3.2 \text { Circular Dichroism Spectroscopy }}$}

To determine the presence and classification of VEGFq and MutVEGF oligonucleotide secondary structures, ODNs were boiled for 5 minutes and annealed by adding physiological buffer containing $20 \mathrm{mM} \mathrm{KH}_{2} \mathrm{PO}_{4}$ dibasic, $120 \mathrm{mM} \mathrm{KCL}, 5 \mathrm{mM}$ $\mathrm{MgCl}_{2}$ then slow cooled to room temperature. Annealed ODNs were diluted in the same buffer to approximately $5 \mu \mathrm{M}$, a concentration which gives an absorbance of 0.800 at 260 $\mathrm{nm}$ according to the spectropolarimeter instructions. Structures of the ODNs in solution were determined by circular dichroism spectroscopy (CD), using a Jasco-810 spectropolarimeter (Jasco). Three scans were performed for each ODN at $25^{\circ} \mathrm{C}$ and then baseline corrected for signal contributions due to buffer. The spectropolarimeter determines the difference between the sample's absorbance of left and right polarized light at each wavelength. To calculate the molar circular dichroism (Molar CD), which 
can be used to determine ODN secondary structure, the absorbance value was divided by a provided system constant, the concentration of the sample, the path length, and the number of bases of the ODN. Each Molar CD value was then plotted versus each corresponding wavelength. These spectra are representative of the average of the scans taken.

\subsection{MTT Assay}

Changes in cell proliferation of lung cancer cell lines and non-transformed fibroblasts in response to VEGFq or MutVEGF were assessed by 3-(4,5-dimethylthiazol2yl)-2,5-diphenyltetrazolium bromide (MTT) assay. Mitochondrial dehydrogenase activity in viable cells metabolizes MTT into formazan crystals. The concentration of formazan, which is directly proportional to cell number, can be quantified spectrophotometrically. A549, H1299, H1944, and Hs27 cells plated in a 96-well plate $\left(1 \mathrm{X} 10^{3}\right.$ cells/well) were treated with $1,3,5,10$, or $15 \mu \mathrm{M}$ of either VEGFq or MutVEGF for $24,48,72,96 \mathrm{~h}$, or 6 days. At the end of the incubation times, $15 \mu \mathrm{L}$ of MTT reagent $(0.1 \mu \mathrm{g} / \mu \mathrm{l}$ phosphate buffered saline (PBS) $)$ was added and cells were incubated at $37^{\circ} \mathrm{C}$ and $5 \% \mathrm{CO}_{2}$. After $4 \mathrm{~h}$, cells were lysed and the formazan product was detected at 570 nm. The optical density of treated wells was standardized to untreated samples in order to quantify the change in proliferation due to ODN treatment.

\subsection{Analysis of VEGFq and MutVEGF Uptake}

Cellular uptake of VEGFq or MutVEGF was analyzed by FACS and confocal microscope image analysis. In order to identify and locate ODNs in these assays, VEGFq 
and MutVEGF were labeled with a 5' fluorescein isothiocyanate (FITC) tag. FITC is a green fluorescent molecule whose excitation max is $493 \mathrm{~nm}$ and emission max is $517 \mathrm{~nm}$. A549 or Hs27 cells were incubated with $10 \mu \mathrm{M}$ of FITC-labeled VEGFq or MutVEGF for 1,24 , or $72 \mathrm{~h}$ before being collected.

For FACS analysis, the cells incubated with FITC-ODNs were first washed with PBS and then resuspended in $1 \mathrm{~mL}$ PBS before being analyzed. The FACS Calibur flow cytometer (BD Biosciences) was calibrated with untreated cells so that on a forward scatter versus side scatter plot, the majority of detected cells had mid-range forward and side scatter. A region was then created that included these cells, which were considered to be intact and viable cells. Those with very low side scatter and/or very low forward scatter were excluded, since these cells were most likely dead cells and/or cellular debris, and would detract from experimental accuracy if included. The cells that fell within the region of interest (ROI) were gated and further analyzed based on their fluorescent intensity. This parameter, which was plotted on a fluorescent intensity histogram, was calibrated so that the untreated cells' peak occurred very low on the scale, ensuring that the curve did not make contact with the y-axis. These calibrations were saved and reused during subsequent experiments for consistency. Each sample was run until 10,000 gated events were collected. The resulting data was analyzed on Flow-Jo (Tree Star, Inc), and the presented histograms are representative of three experiments.

For confocal microscopy, Hs27 or A549 cells (grown in 2-well chambered slides) were treated for $72 \mathrm{~h}$ with FITC-labeled VEGFq or MutVEGF. Slides were washed with PBS, and fixed in 4\% paraformaldehyde for 20 min before being washed with PBS again. Cell nuclei were stained with 4',6-diamidino-2-phenylindole (DAPI), a blue fluorescent 
molecule whose excitation max is $358 \mathrm{~nm}$ and emission max is $461 \mathrm{~nm}$. Following another wash with PBS, slides were mounted with Prolong Gold (Invitrogen) anti-fade reagent to preserve fluorescence. Cells were visualized by confocal microscopy with an Olympus Fluoview FV500 (Olympus) laser scanning microscope. Prior to image capture, laser intensity and gain were optimized and permanently set for FITC and DAPI in order to minimize background noise while maximizing fluorescent signal without losing accuracy and precision due to too high of a signal intensity. Samples were visualized at $60 \mathrm{X}$ magnification and sequentially scanned with the two lasers to minimize spectral crossover. The obtained images of FITC only, DAPI only, bright field only and fluorescent overlay were compiled, and a representative set of each sample was selected.

\section{$\underline{3.5 \text { Cell Cycle Analysis }}$}

A549 or Hs27 cells treated for $24,48,72$, or $96 \mathrm{~h}$ with $10 \mu \mathrm{M}$ VEGFq or MutVEGF were collected and washed in PBS. Cells were processed with the CycleTest $^{\mathrm{TM}}$ Plus DNA Reagent kit (BD Biosciences), during which they were lysed with trypsin in spermine teterahydrochloride detergent buffer and the isolated nuclei were stained with propidium iodide. Prepared nuclei were then analyzed by FACS to determine the distribution and percentage of cells in each cell cycle phase. For calibration, similar gating was applied as in the ODN uptake experiments to collect only the cells with mid-range side and forward scatter. A histogram of fluorescent intensity was created from 10,000 gated cells. Results were analyzed with Flow-Jo, and the spectrum provided is a representative of three cell cycle experiments at the $72 \mathrm{~h}$ time point. 


\subsection{Serum and Intracellular Stability of VEGFq}

VEGFq and MutVEGF sequences were radiolabeled using $\left[\gamma_{-}{ }^{32} \mathrm{P}\right]$-dATP with $\mathrm{T}_{4}$ polynucleotide kinase (Invitrogen) and incubated at $10 \mu \mathrm{M}$ in DMEM medium with $10 \%$ FBS or in the presence of A549 cytoplasmic (S100) or nuclear extract at $37{ }^{\circ} \mathrm{C}$ for $0,1,2$, 4, 24, 48, 72, or $96 \mathrm{~h}$. After heating in $98 \%$ formamide buffer to $65^{\circ} \mathrm{C}$, ODNs were run on a $12 \%$ denaturing gel and imaged by autoradiography. The obtained images are representative of three stability experiments.

\subsection{Western Blot analysis}

To determine the changes in protein expression, A549 cells were incubated with $10 \mu \mathrm{M}$ of VEGFq or MutVEGF for 24, 72, 96, or $144 \mathrm{~h}$ before being collected and lysed. Samples' total protein concentrations were determined by Lowry Assay calibrated with bovine serum albumin (BSA). Equal quantities of A549 total cell lysates were separated by $4-15 \%$ SDS-Tris polyacrylamide gel electrophoresis (SDS-PAGE) and electroblotted onto PVDF membranes. Following transfer, membranes were air dried and then re-wetted with $100 \%$ methanol. Successful electrophoresis and protein transfer was confirmed by staining with Ponceau S dye. In order to reduce nonspecific binding and background signal, the membranes were blocked in 5\% milk in a solution of $0.05 \%$ Tween-20 in PBS for $1 \mathrm{hr}$ at room temperature with agitation. Membranes were then incubated with agitation overnight at $4{ }^{\circ} \mathrm{C}$ with VEGF, ERK1/2, p-ERK1/2, AKT1/2/3, p-AKT1/2/3, or FLK-1 antibodies (Santa Cruz Biotechnology) diluted in the blocking solution. After washing three times with $0.05 \%$ Tween-20 in PBS for 5 minutes at room temperature, the membranes were incubated with a horseradish peroxidase (HRP)-conjugated secondary 
antibody diluted in the blocking solution. Following another set of three washes with PBS-Tween, an additional wash for 5 minutes at room temperature was performed to remove the detergent. Proteins were visualized by standard chemiluminescence (ECL) methods (GE Healthcare). All films were scanned with an optical scanner (Epson Expression 1680) and band densities were quantified using UNSCAN-IT software (Silk Scientific).

Equal loading of protein samples was verified by probing the membrane with a mouse monoclonal anti- $\beta$-actin primary antibody or a mouse monoclonal anti-GAPDH primary antibody (Santa Cruz Biotechnology). Prior to re-probing, membranes were incubated with Restore Western Blot Stripping Buffer (Thermo Scientific) for 15 minutes at room temperature with agitation and washed with PBS to remove the previously bound antibodies. To correct for possible unequal loading, each band density was normalized to its respective $\beta$-actin or GAPDH density.

\section{$\underline{3.8 \text { p-FLK-1 ELISA }}$}

To quantify the changes in phosphorylation of VEGFR-2, A549 cells were incubated with $10 \mu \mathrm{M}$ of VEGFq or MutVEGF for $72 \mathrm{~h}$ before being collected and lysed. Samples' total protein concentrations were determined by Lowry Assay calibrated with BSA. The sandwich ELISA method (R\&D Systems) was used to examine 25 and $50 \mu \mathrm{g}$ amounts of A549 total cell lysates, according to kit instructions. A 96-well microplate was incubated overnight at room temperature with a phospho-FLK-1 capture antibody and washed 5 times with $0.05 \%$ Tween-20 in PBS before being blocked with a $1 \%$ BSA in PBS solution for $2 \mathrm{~h}$ at room temperature. Following another round of washes, samples 
were diluted to 25 or $50 \mu \mathrm{g} / 100 \mu \mathrm{L}$ in a provided dilution buffer and incubated along with 4 and $8 \mathrm{ng} / \mathrm{mL}$ of a provided phospho-FLK-1 control in separate wells for $2 \mathrm{~h}$ at room temperature. Following another washing step, the plate was incubated with a phospho-FLK-1 HRP-conjugated detection antibody for $2 \mathrm{~h}$ at room temperature. After a final set of washing, the substrate solution was added, and following a 20 min room temperature incubation, the stop solution. The resulting absorption was read at $450 \mathrm{~nm}$. Expression levels were compared to untreated samples to calculate a percent of untreated. The obtained figure is representative of an average of three experiments.

\subsection{Boyden Chamber Invasion/Migration Assay}

The Boyden chamber assay was used to assess the metastatic potential of A549 cells in response to VEGFq or MutVEGF. A549 cells pretreated with $10 \mu \mathrm{M}$ VEGFq or MutVEGF for $72 \mathrm{~h}$ were detached with TripleE (Invitrogen) and seeded $\left(5 \mathrm{X} 10^{5} / \mathrm{mL}\right)$ in serum-free medium in the top side of the chamber containing an $8 \mu \mathrm{m}$ pore polycarbonate filter coated with Matrigel or a control chamber without Matrigel (BD Biosciences). Medium containing 10\% FBS was applied to the lower chamber as a chemoattractant. VEGFq or MutVEGF $(10 \mu \mathrm{M})$ were added into the serum-free medium in the top chamber and the cells were incubated for $24 \mathrm{~h}$ at $37^{\circ} \mathrm{C}$. At the end of the incubation, cells in the upper surface of the membrane were carefully removed with a cotton swab and the cells that had invaded across the Matrigel to the lower surface of the membrane were fixed with 100\% methanol for 2 min, briefly rinsed in PBS, and stained with crystal violet stain (Sigma-Aldrich) for 2 min. Excess stain was removed by washing in $\mathrm{dH}_{2} \mathrm{O}$ before drying overnight at room temperature. Each insert was then imaged at $20 \mathrm{X}$ 
magnification using a microscope, and the number of cells present was physically counted. The percentage of cells that invaded through the Matrigel and migrated through the filter (percent invasion) was calculated by dividing the number of cells present in each treatment's Matrigel chamber by the number of cells present in its respective control chamber. The invasion index was then calculated by dividing the percent invasion of the treated cells by the untreated percent invasion.

\subsection{VEGFq In Vivo Activity}

To determine the in vivo effect of VEGFq on cell proliferation of NSCLC xenografted in nude mice, $10 \times 10^{6}$ A549 cells suspended in $100 \mu \mathrm{L}$ PBS were injected subcutaneously in each mouse's left dorsal flank and grown for 10 days. After tumors became visible under the skin, mice were sorted into groups of small, medium, and large tumors based on visual inspection of tumor sizes. An equal number of each size set was then sorted into either the control or treatment groups. The treatment group was then injected intraperitoneally with $10 \mathrm{mg} / \mathrm{kg}$ VEGFq dissolved in an equal volume of $2 \mathrm{X}$ RPMI media for a final volume of $100 \mu \mathrm{L}$ every weekday until tumor sizes reached a large enough size that required sacrificing.

Tumor length and width measurements for all animals were collected every other weekday during the study. The tumor volumes at each time point were calculated by multiplying the length measurement by the width measurement by one half of the width measurement. To correct for the differences in beginning tumor sizes, the percent change in growth was calculated for each animal by dividing the difference between each 
measurement and the initial measurement by the initial measurement, and then averaged for each group.

\subsection{VEGFq In Vivo Stability and Biodistribution}

VEGFq stability and biodistribution in the in vivo NSCLC xenograft nude mouse model was determined by intraorbital injection of $1 \mathrm{mg} / \mathrm{kg}$ of Alexa Fluor 750 labeled VEGFq mixed with an equal volume of $1 \mathrm{X}$ RPMI media to a $100 \mu \mathrm{L}$ final volume for subsequent imaging. Non-injected control animals were used to establish a baseline for no fluorescent signal. Treatment and control animals were sedated with isoflurane and imaged simultaneously in a Photo Imager RT system immediately after injection as well as at $1,2,6,24,48$, and 72 hour time points. Detector settings were optimized to minimize background noise while maximizing signal. To quantify the signal coming from only the tumors, ROIs of equal area that encompassed both the treatment and control animals' tumors were selected and a signal strength value was collected. To eliminate the effect of noise variation on the results, the fluorescence intensity of the control ROI was subtracted from that of the treatment ROI. This calculation provided a value for the number of photons per square centimeter due to fluorescently tagged 750-labeled VEGFq alone being emitted from the tumor due to treatment with VEGFq. These values were plotted as a function of time to determine the extent of oligonucleotide retention over time. 


\section{RESULTS AND DISCUSSION OF RESULTS}

4.1 VEGFq forms a parallel quadruplex in solution

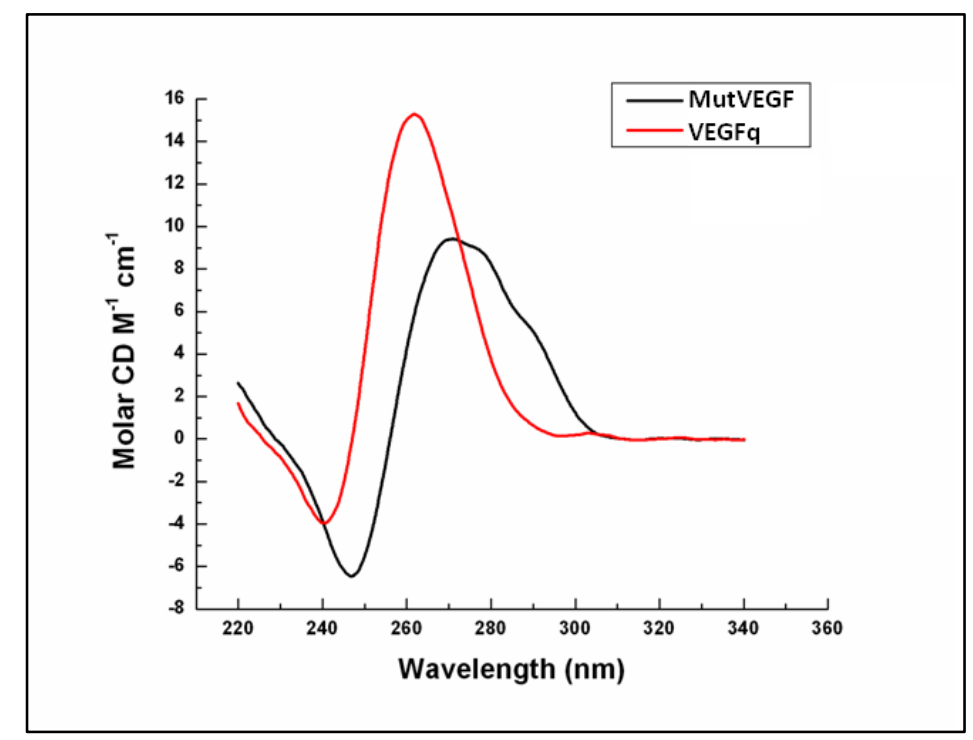

FIGURE 1 - Representative CD scan of the quadruplex forming oligonucleotide VEGFq and the non-quadruplex forming oligonucleotide MutVEGF.

To characterize the secondary structure of VEGFq and MutVEGF, oligonucleotides were analyzed using Circular Dichroism spectroscopy, a technique that has been used to examine the secondary structures of nucleic acids in solution for several decades (Gray, 2003). VEGFq dissolved in physiological solution had a peak absorbance at $260 \mathrm{~nm}$ and a trough absorbance at $240 \mathrm{~nm}$, indicative of parallel quadruplex formation (Figure 1). MutVEGF did not form a stable parallel quadruplex in solution, which was reflected by a shift in its spectrum (Rankin, 2005).

These results confirm that VEGFq can be examined as a quadruplex forming oligonucleotide, and whose effects can be attributed to this characteristic. Additionally, 
MutVEGF, which arises from the mutation of the VEGFq sequence so that did not have three adjacent guanines in the sequence, is inhibited from forming a quadruplex; therefore, it can be used as a negative quadruplex control for VEGFq.

\subsection{VEGFq inhibits NSCLC cell proliferation}

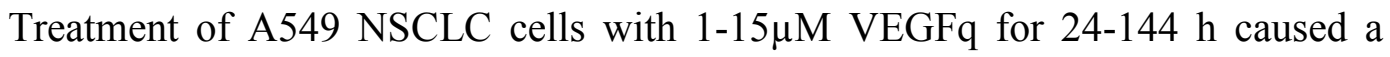
dose and time-dependent decrease in proliferation, culminating to $80 \%$ inhibition with 10 $\mu \mathrm{M}$ and $90 \%$ inhibition with $15 \mu \mathrm{M}$ after 6 days compared to untreated cells with an $\mathrm{IC}_{50}$ of less than $5 \mu \mathrm{M}$ (Figure 2A). Little change in proliferation occurred with MutVEGFq (Figure 2B). The effect of dose escalation of VEGFq on cell proliferation was also examined in H1299 (Figure 2C) and H1944 NSCLC cell lines after 72 and 144 h. There was a significant dose-dependent decrease in cell proliferation with $\mathrm{IC}_{50}$ values of less than $15 \mu \mathrm{M}$ after $144 \mathrm{~h}$; however, both cell types were slightly less sensitive than A549 cells. Since the growth arrest occured in the VEGFq treatment groups and not the MutVEGF groups, these observations suggest that the quadruplex-forming nature of VEGFq results in decreased cell growth of NSCLC.

Alternatively, treatment of Hs27 non-transformed human fibroblast cells with VEGFq caused no significant change in cell proliferation (Figure 2D) as compared to A549 cells treated in parallel. MutVEGF did not affect the growth of either cell line. These results suggest that growth inhibition due to VEGFq is specific to cancer cells as compared to normal cells. 

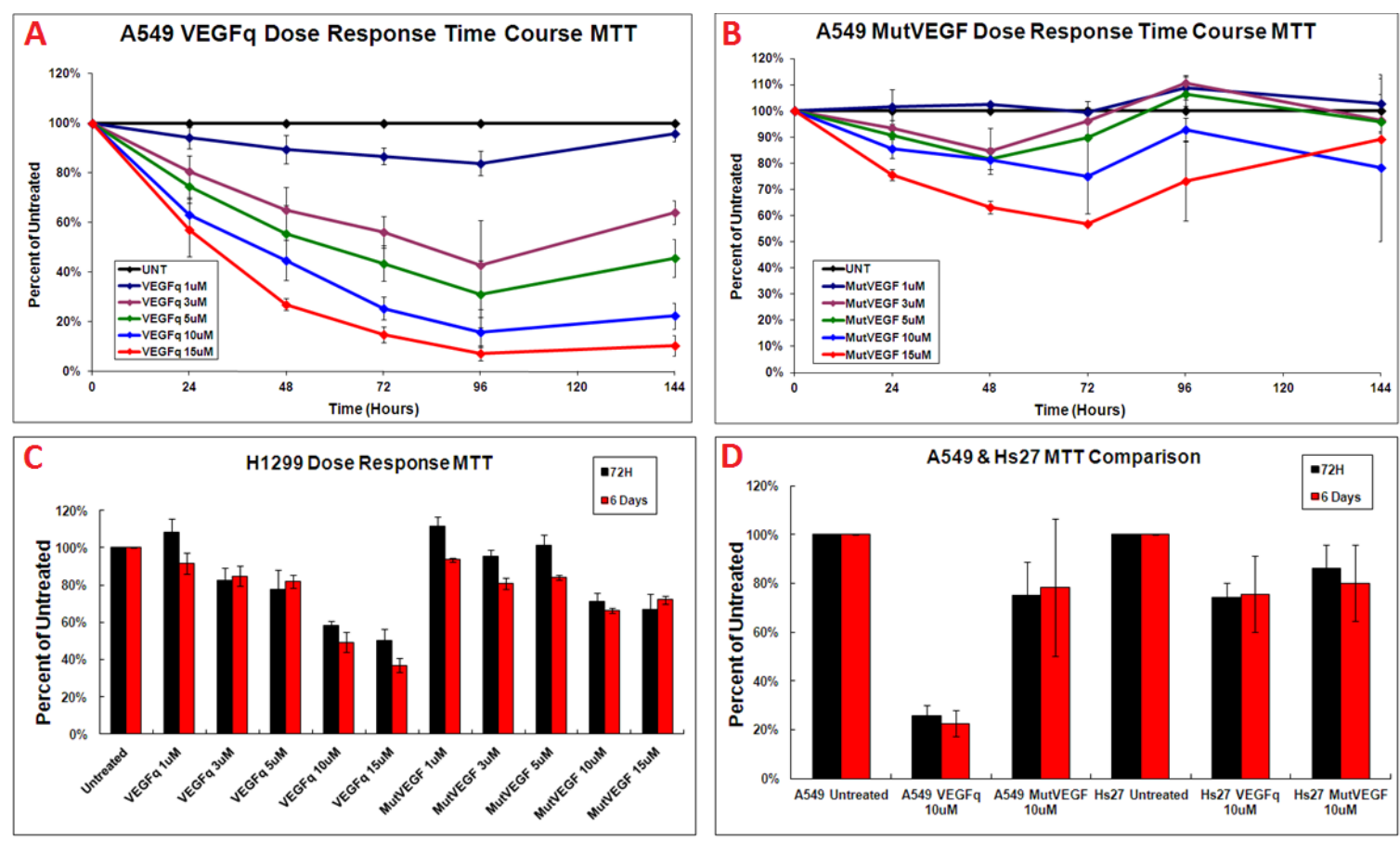

FIGURE 2 - MTT dose response time courses for VEGFq and MutVEGF in A549, H1299, and Hs27 cells.

\subsection{Enhanced cellular uptake of VEGFq}

FITC-VEGFq was immediately taken into cells after only 1 hour of treatment, with enhanced uptake after 24 hours and retained signal at 72 hours post-treatment (Figure 3A). Significantly less FITC-MutVEGF appeared to be taken into cells at all time points. Confocal microscopy confirmed these results, showing both cytoplasmic and nuclear localization of FITC-VEGFq and almost no uptake of MutVEGF at the same intensity settings (Figure 3B). These results suggest that VEGFq is greatly internalized by A549 cells compared to MutVEGF and largely located in the nucleus and cytoplasm. A key improvement of VEGFq upon traditional antisense oligonucleotide therapies is exhibited here; while antisense oligonucleotides are typically linear and have low cellular 
uptake, the quadruplex secondary structure exhibited by VEGFq may cause the observed cellular uptake enhancement (Kurreck, 2003).
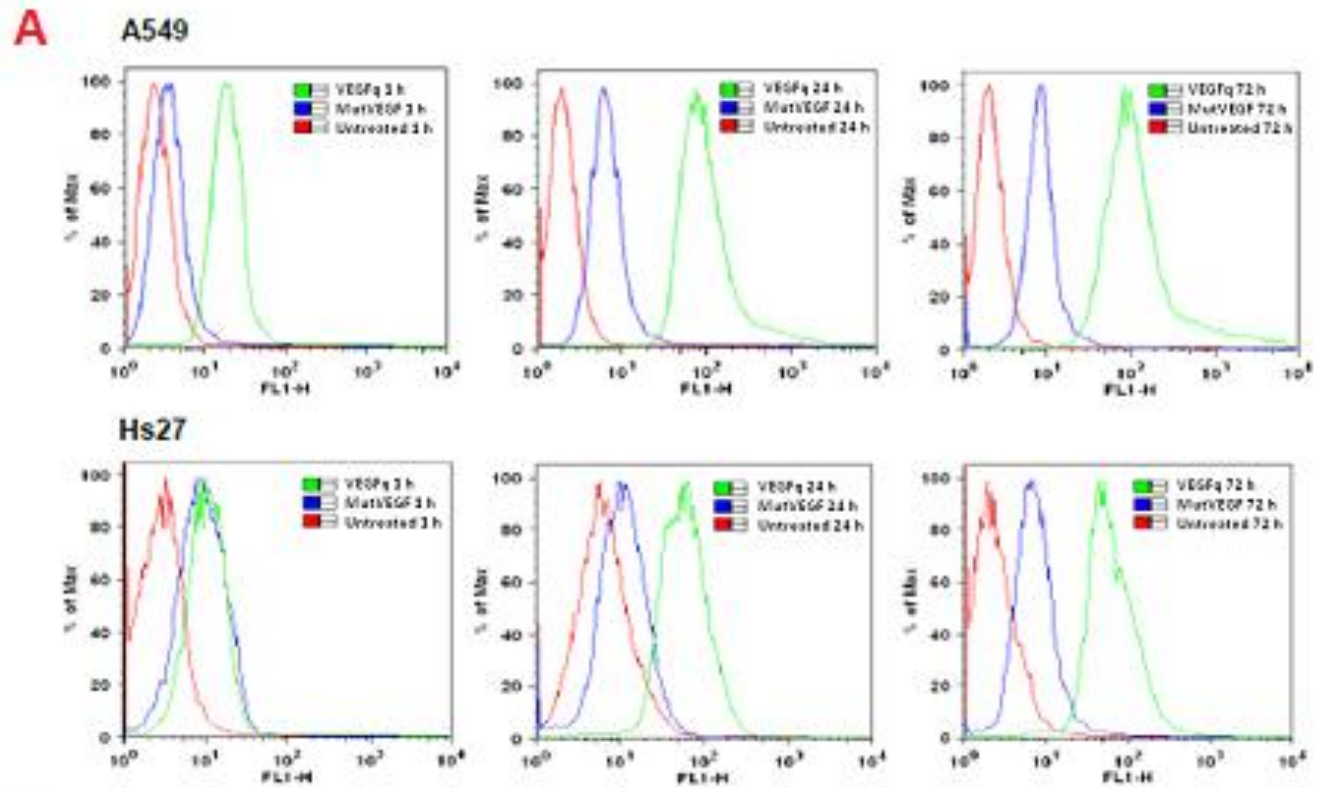

B

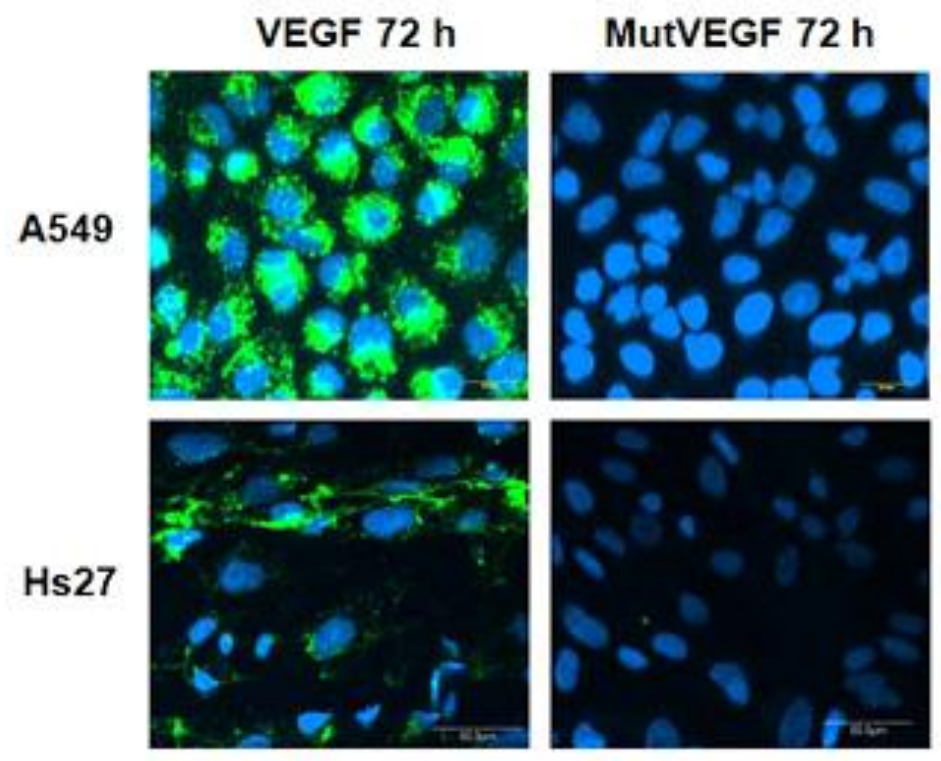

FIGURE 3 - Cellular uptake and localization of VEGFq and MutVEGF in A549 and Hs27 cells. 
Similarly, non-transformed Hs27 cells also took up FITC-VEGFq after 1 hour, with greater uptake over time, which appeared more largely localized in the cytoplasm. Uptake of VEGFq into Hs27 cells suggest that the anti-proliferative effect of VEGFq on A549 cells is unrelated to its ability to be taken into cells, but instead may be cancer specific.

\subsection{VEGFq and MutVEGF do not cause a change in cell cycle}

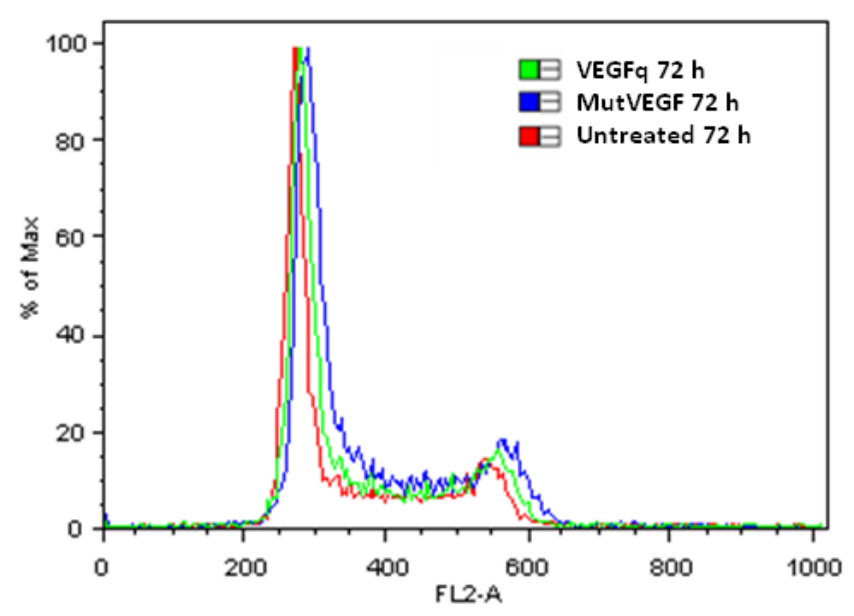

FIGURE 4 - Cell cycle analysis of A549 cells treated with VEGFq or MutVEGF.

To determine if treatment of A549 cells with VEGFq or MutVEGF caused an

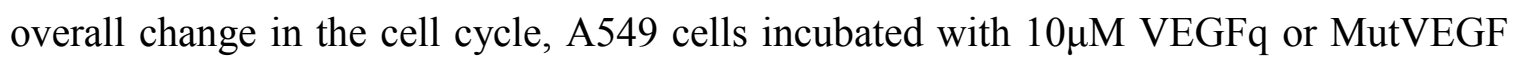
for 24-96 $\mathrm{h}$ were assessed. No significant change in the cell cycle occurred with VEGFq or MutVEGF treatment (Figure 4). A representative figure at $72 \mathrm{~h}$ is shown; however, cell cycles at all time points showed similar results. 


\section{$\underline{4.5 \text { Enhanced VEFGq serum and cytoplasmic stability }}$}

Since the therapeutic utility of ODNs is limited by their susceptibility to nuclease degradation, the stability of VEGFq was examined in serum and intracellularly. ${ }^{32} \mathrm{P}-$ labeled VEGFq was inherently stable in serum and in the presence of nuclear and cytoplasmic extracts, while MutVEGF almost immediately degraded into a smaller

product (Figure 5). This remarkable increase in VEGFq stability is likely related to the inherent nuclease resistance of quadruplex DNA.

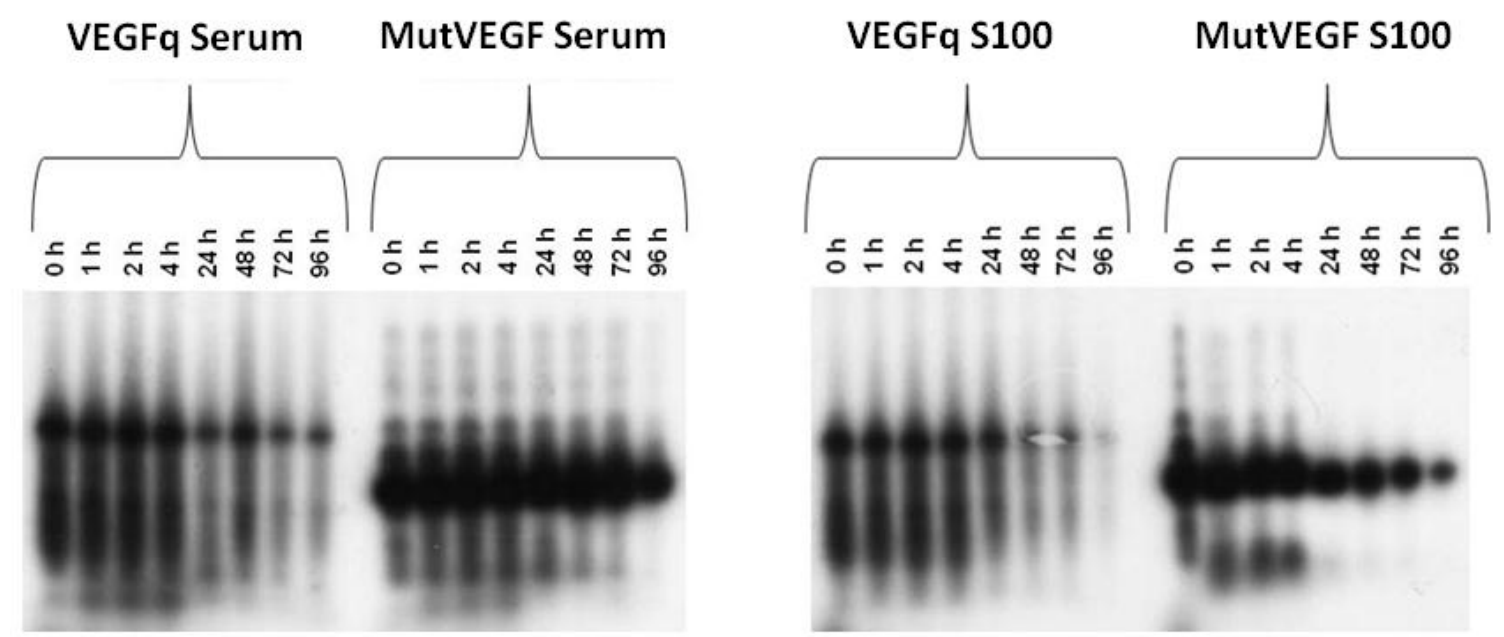

FIGURE 5 - Comparison of VEGFq and MutVEGF stability in serum and in cell extracts.

\section{$\underline{4.6 \text { VEGFq treatment decreases VEGF protein levels }}$}

To determine if VEGFq decreases VEGF protein levels, A549 cells were treated with $10 \mu \mathrm{M}$ VEGFq or MutVEGF and analyzed by Western Blotting. Treatment of A549 cells with VEGFq did not decrease VEGF protein levels after 24 and $72 \mathrm{~h}$, however 
caused a marked decrease in expression $(<60 \%$ of untreated) after $96 \mathrm{~h}$ and 6 days. MutVEGF treatment did not alter VEGF protein expression levels (Figure 6).

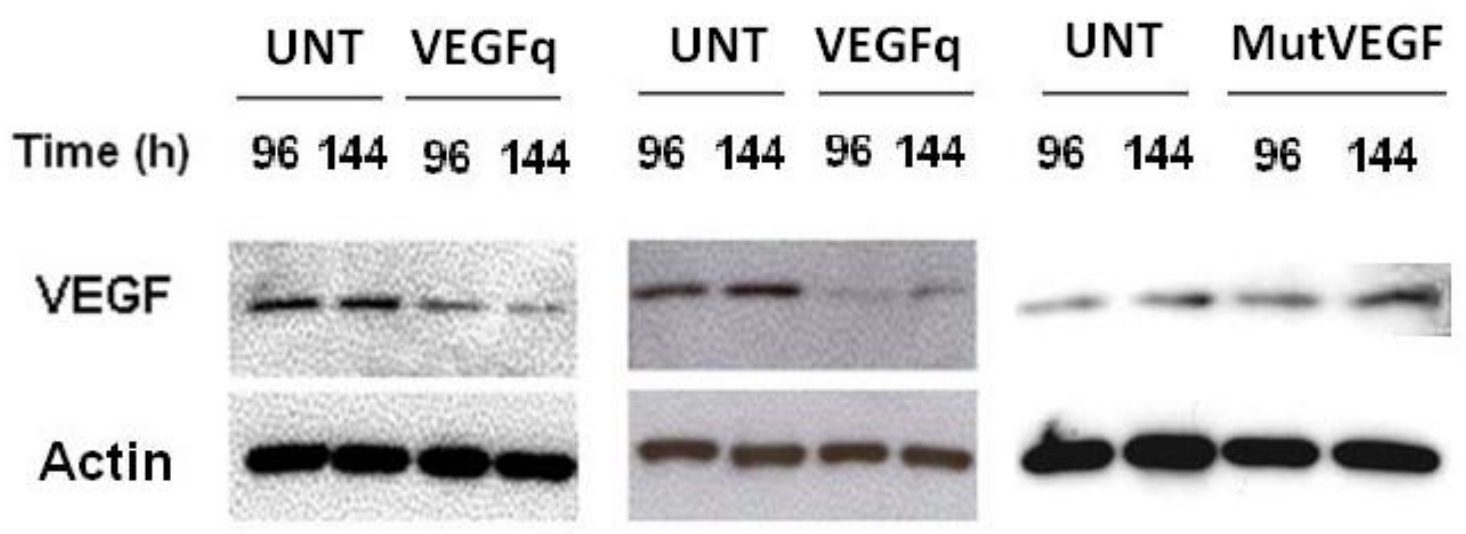

FIGURE 6 - Western Blot results of VEGFq and MutVEGF treated A549 cells.

\subsection{VEGFq alters activation of FLK-1 and downstream signaling}

Decreased VEGF protein resulting from VEGFq treatment corresponded with a small decrease in FLK-1 phosphorylation after $72 \mathrm{~h}$ compared to untreated A549 cells, as determined by ELISA analysis (Figure 7C). A less significant change in FLK-1 phosphorylation occurred with MutVEGF treatment. However, total FLK-1 expression remained constant after either VEGFq or MutVEGF treatment (Figure 7B).

VEGFq treatment of A549 cells slightly decreased total ERK 1/2 levels and markedly decreased p-ERK 1/2 (p42/p44), beginning at $72 \mathrm{~h}$ and continuing thorough 96 h (Figure 7D). Although Akt total 1/2/3 levels remained relatively constant, p-Akt 1/2/3 levels were also significantly decreased after 72 and $96 \mathrm{~h}$ by VEGFq (Figure 7E). These results suggest that the change in phosphorylation of FLK-1 may be due to a decrease in 
binding of VEGF to FLK-1, causing a subsequent decreased in the activity of downstream kinases ERK and Akt.
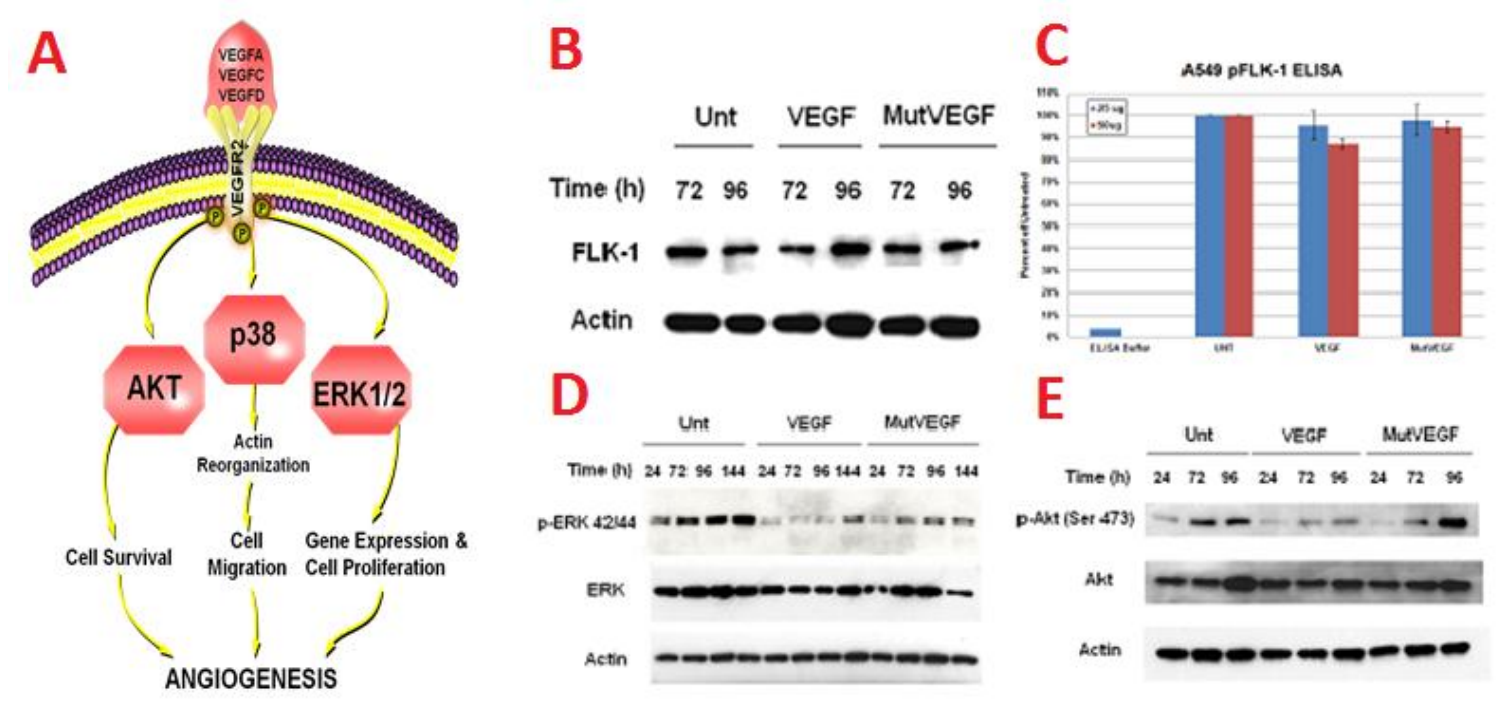

FIGURE 7 - Schematic of VEGFR-2 signaling and Western Blot and ELISA analysis of changes in receptor and downstream protein and phosphorylation levels due to VEGFq and MutVEGF treatment in A549 cells.

\subsection{VEGFq inhibits NSCLC cell invasion}

To determine whether decreased VEGF from VEGFq treatment changed A549 cell invasion, cells treated with $10 \mu \mathrm{M}$ VEGFq for $24 \mathrm{~h}$ were evaluated by Boyden chamber analysis. Compared to untreated, VEGFq decreased A549 cell invasion by $40 \%$, while MutVEGF had no effect (Figure 8). There were no changes in cell number of the control plates in response to VEGFq or MutVEGF treatment. 


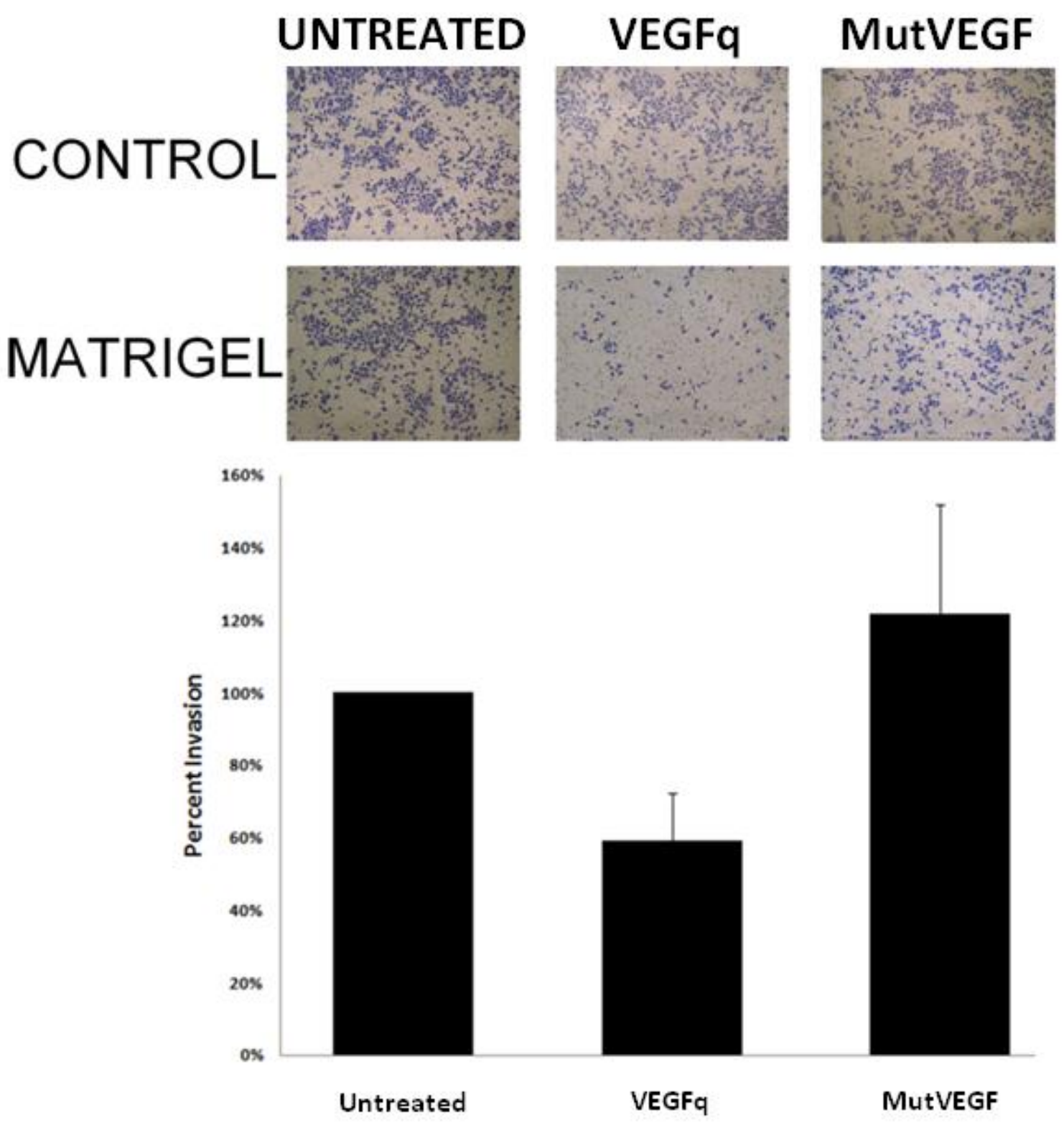

FIGURE 8 - Invasion/Migration analysis of A549 cells treated with VEGFq or MutVEGF.

4.9 VEGFq inhibits in vivo NSCLC cell proliferation

Following the successful xenografting of A549 cells, nude mice were injected daily for 14 weekdays with VEGFq, to examine possible growth hindering effects in vivo. 
Even after only two days of treatment, the VEGFq animals' tumor growth was significantly arrested compared to the untreated arm (Figure 9). The significant difference between arms was continued throughout the entire experiment. These results suggest that VEGFq treatment can inhibit NSCLC tumor growth in vivo.

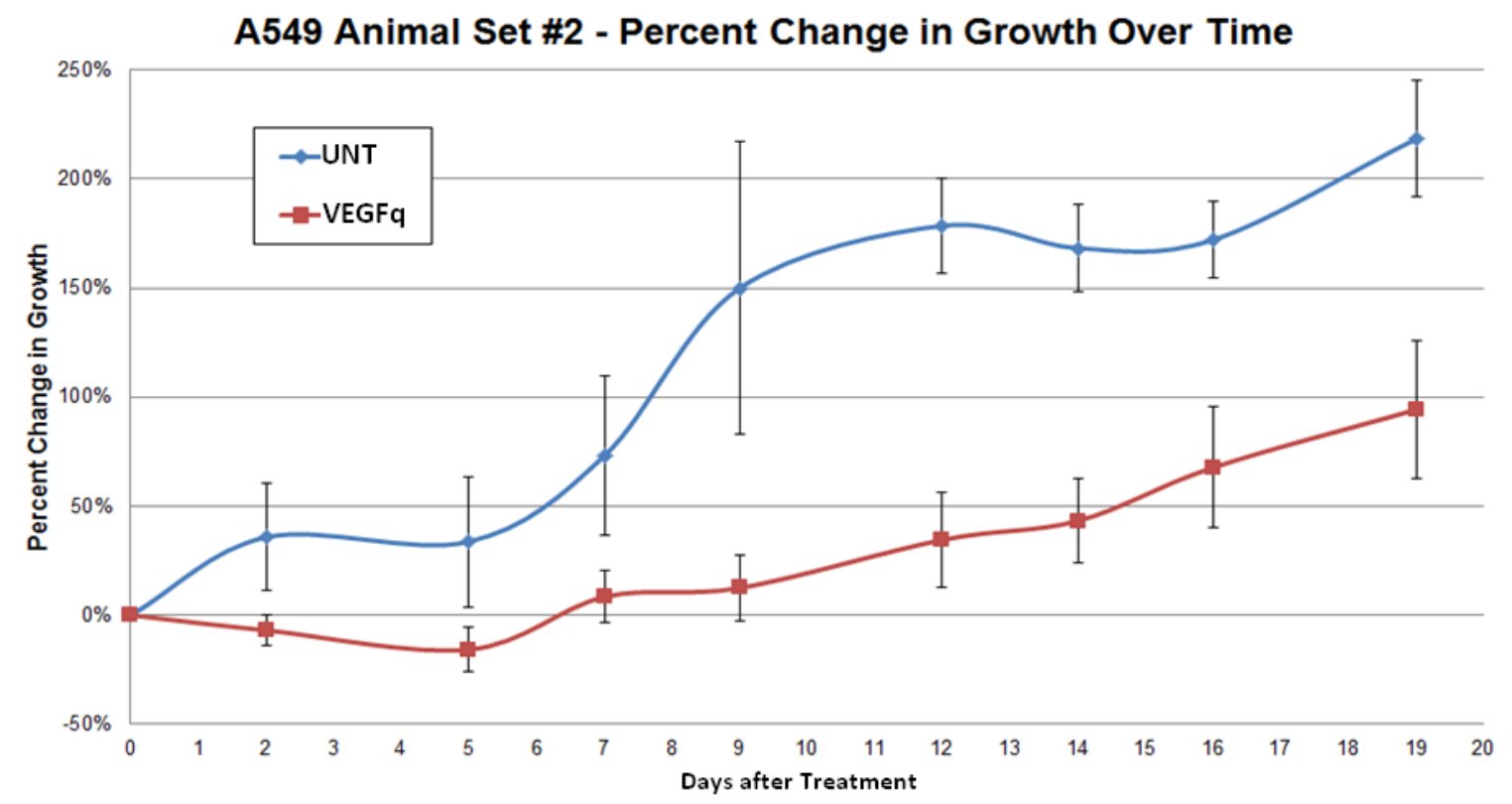

FIGURE 9 - In Vivo change in tumor growth of xenografted A549 cells treated with VEGFq or MutVEGF.

\section{$\underline{4.10 \text { Enhanced VEGFq in vivo stability and tumor localization }}$}

To determine the stability and biodistribution of VEGFq in vivo, A549 cells were xenografted into nude mice and were imaged following an injection of VEGFq tagged with Alexa Fluor 750. Subsequent quantitation of the fluorescent intensity was performed to yield a photons $/ \mathrm{cm}^{2}$ value. In order to eliminate background noise, the fluorescence signal (photons $/$ second $/ \mathrm{cm}^{2}$ ) of the untreated mouse's tumor was subtracted from that of the mouse treated with VEGFq. This value was multiplied by the acquisition time (4 
seconds), providing a fluorescent intensity per unit area value (photons $/ \mathrm{cm}^{2}$ ). If an oligonucleotide calibration curve could be created, the fluorescent intensity value could be used to calculate the concentration of oligonucleotide delivered to the tumor. Results showed striking uptake of VEGFq into the tumor regions and prolonged storage of oligonucleotide in the tumor at $72 \mathrm{~h}$ after treatment (Figure 10).

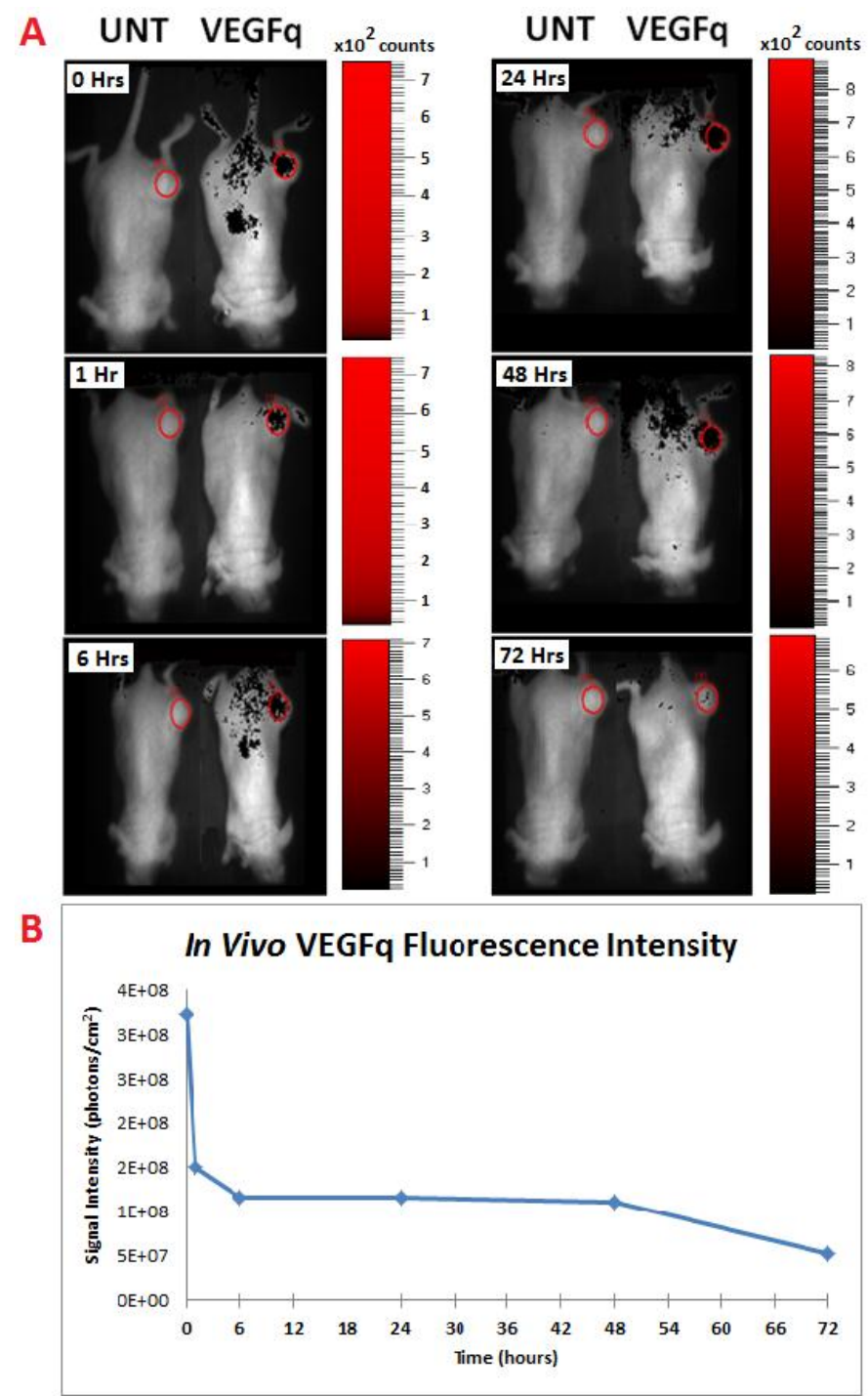

FIGURE 10 - In Vivo Alexa Fluor 750 labeled VEGFq fluorescent intensity in xenografted A549 tumors. 


\section{CONCLUSIONS}

The following list of conclusions is based upon on the results and analysis of the experiments performed during this study.

- VEGFq forms a stable parallel quadruplex structure under physiological conditions.

- VEGFq significantly inhibits NSCLC cell growth not due to cell cycle arrest with an IC50 of less than $5 \mu \mathrm{M}$ in A549 cells and an IC50 $<10 \mu \mathrm{M}$ with H1299 and H1944 cells. It does not inhibit non-transformed cell growth.

- Inhibition of cell growth by VEGFq is not due to changes in the cell cycle.

- NSCLC cell invasion is decreased by $40 \%$ through treatment with VEGFq.

- VEGFq is taken up into cells without transfection enhancement as early as 1 hour, localizing in both the nucleus and cytoplasm.

- VEGFq is highly stable in serum and intracellularly for longer than 96 hours.

- VEGFq treatment of NSCLC cells downregulates VEGF protein expression, decreasing activation of FLK-1 corresponding with decreased ERK and AKT phosphorylation.

- VEGFq causes a significant decrease in NSCLC cell growth in vivo.

- VEGFq is highly stable and remains in NSCLC xenograft tumors for greater than 72 hours.

- VEGFq preferentially localizes in xenograft tumors compared to other tissues. 


\section{RECOMMENDATIONS}

Although the research performed has answered many questions regarding VEGFq and its biological significance, there are an equal number that have arisen through the completion of this study. Below is a list of recommendations for future experiments that will further the knowledge of the quadruplex-forming oligonucleotide field, especially with regards to VEGFq.

First and foremost, although it is known that it is readily taken up into cells, the exact mechanism of this process remains uncharacterized. Since there is more VEGFq taken up in comparison to MutVEGF, this suggests that its quadruplex secondary structure may play a role in its uptake. By studying VEGFq's uptake in the presence of a quadruplex inhibitor, it can be determined if the quadruplex indeed makes it more favorable for uptake into cells. Additionally, since its large molecular weight and highly polar nature makes VEGFq thermodynamically impossible for passive diffusion to take place, the discovery of either a transport protein or receptor that would facilitate its transport or endocytosis would reveal a logical procedure for this process. A third method of uptake could possibly involve VEGFq binding to a carrier protein that is then transported across the plasma membrane and then disassociates from VEGFq, freeing the oligonucleotide once inside. Discovering this uptake mechanism will not only shed light on how quadruplex-forming oligonucleotides get inside cells to such a high degree, but it could also teach us more about how they interact with other biomolecules.

Secondly, although it is hypothesized that VEGFq binds exclusively to the VEGF promoter, physical evidence is still lacking that would solidify this claim. A mere 
decrease in VEGF protein expression in response to VEGFq treatment does not necessarily prove that it binds to the promoter sequence. RT-PCR analysis of RNA extracted from untreated and VEGFq treated cells could be used to examine the changes in VEGF expression that may indicate that VEGFq's effects are at the transcriptional level. Another possible method of determining VEGFq's transcriptional effects is known as permanganate genomic footprinting. This method utilizes the ability of permanganate $\left(\mathrm{MnO}_{4}{ }^{-}\right)$to specifically oxidize thymines in single stranded DNA, namely those present in the transcription bubbles created by RNA polymerases. These oxidized thymines are cleaved with piperidine, the resulting fragments are amplified using PCR primers specific to the gene of interest, and the PCR product is run on a gel and visualized using a nucleic acid stain such as ethidium bromide (Gilmour, 2009). The resulting bands indicate the locations of transcriptions bubbles and subsequently, the locations of RNA polymerases on a given gene. If the polymerases on an active gene are free to proceed, it is expected that there will be a large number of band sizes signifying the protein's progression along the gene; however, VEGFq treated samples are expected to exhibit very few, short bands, since quadruplex formation would inhibit the polymerase to proceed, indicating that VEGFq inhibits transcription of the VEGF gene.

VEGFq could be acting through alternative mechanisms than its proposed binding to the VEGF promoter, such as antisense binding to VEGF transcripts or through binding to VEGF protein. It may prove to be important to elucidate VEGFq's exact mechanism of action to fully understand its effects. Performing an experiment similar to ChIP (chromatin immunoprecipitation) where cell extracts are incubated with VEGFq- 
modified magnetic or agarose beads followed by mass spectrometry for proteins or sequencing for nucleic acids, may identify the binding targets of VEGFq.

Global changes in gene expression and polymerase locations due to treatment with VEGFq can be determined by the utilization of next generation sequencing following the above described methods (RNA extraction, permanganate footprinting, and ChIP). Since the completion of the human genome project, the exact sequence of our DNA and the locations and sequences of all genes have been identified; therefore, through the use of next generation sequencing, extracted RNA or DNA can be sequenced and aligned to their exact locations in the genome, essentially determining their identities. This data can be used to identify the basal levels and changes due to treatment across the entire genome. This process can be helpful to interrogate genes that would not normally be examined with standard single gene approaches.

RNA sequencing can also be enhanced to allow for quantitation of the number of transcripts per cell through the use of synthetic spike-in RNA's. These synthetic RNA's are comprised of specific sequences that are not present in our genome, so they will not be aligned to any gene, but rather they will be identified separately. If the sample's cell number is determined prior to RNA extraction, a known number of copies of spike-in RNA can be added to each sample prior to library construction, which will allow for the standardization of the sequencing process across samples as well as a calculation for the number of copies of each transcript per cell. Finally, comparing the input and sequenced spike-in RNA copies can also serve as a sequencing efficiency check, since the two copy numbers should be equal. Although the practice of synthetic spike-in RNA's is relatively new, it is clear that there are many advantages to using them (Jiang, 2011). 
The author proposes that an undeveloped procedure that combines two established methods may prove to be the best way to answer the questions surrounding VEGFq's interactions. A method known as dimethyl sulfate (DMS) footprinting can be used to mark the open N7 position of both single stranded and duplex guanines, but not guanines associated with a neighboring guanine involved in a G-quartet where the N7 is engaged in a hydrogen bond. Marked bases are then susceptible to heating, and following a mild alkali treatment, creating fragments that are involved in G-quartets (Neidle, 2006). While this may reveal where a quadruplex forms (most notably at the VEGF promoter), it does not prove that the VEGFq that was added to cells is the one associated with the endogenous sequence, but simply its treatment induced some sort of quadruplex formation. Therefore, another method, known as Forster Resonance Energy Transfer (FRET), is necessary to further examine this interaction. This method utilizes the quantum phenomenon between two fluorophores where, when the donor and acceptor molecules are in close enough proximity (within $10 \mathrm{~nm}$ ), the excitation of the donor results in the emission of the acceptor (Didenko, 2001) that can be visualized using standard fluorescence detection methods. Additionally, if standards of known of fluorescence and varying ODN concentrations per cell number are used, the FRET signal can be quantified and the kinetics and efficiency of quadruplex formation can be determined. Prior to the addition of VEGFq, extracted DNA can be fragmented (to increase PCR efficiency) and guanines on one strand can be marked through one round of PCR with fluorescently labeled dGTP added rather than normal dGTP. The PCR product can then be incubated with FITC-labeled VEGFq, then undergo DMS treatment before FRET detection. As long as the modified guanines do not inhibit quartet formation, this 
process will essentially determine if VEGFq can bind with an endogenous sequence to form a quadruplex. Additionally, if the quadruplexes positive for FRET can somehow be purified, these DNA fragments can be sequenced to determine their sequence and then can be mapped to reveal their exact location in the genome. While the usage of FRET without the DMS step may reveal other binding locations of VEGFq, the elimination of non-quartet DNA may decrease the chance of noise due to non-specific binding. NonDMS FRET should also be examined with other molecules modified with fluorophores, notably those identified in the ChIP experiments, to determine the cell-wide interactions of VEGFq.

Thirdly, chemotherapy drugs and other quadruplex-forming oligonucleotides that target other genes should be tested in combination to VEGFq to identify any sets that exhibit synergy. Synergy is when the effect caused by the grouping of two or more agents is greater than the sum of the effects of the individual agents. Multiple chemotherapies are often prescribed to patients because they are synergistic and therefore work against the cancer better together than a single agent alone. If $\mathrm{VEGFq}$ is found to have synergistic effects with other drugs, it would enhance its efficacy, versatility, and marketability as an anti-cancer agent.

Lastly, as nanotechnology continues to be developed, it is evident that it can be useful in many fields, including medicine. If VEGFq could be coupled with nanoparticles, such as exosomes, it is hypothesized that its efficiency would be enhanced. Not only could uptake into tumors be enhanced, but targeting to tissue types would allow for less waste of oligonucleotides that end up in normal cells. In fact, it has been suggested that exosomes are capable of crossing the blood brain barrier. This would 
allow VEGFq to be delivered for the treatment of brain cancer, another disease in which VEGF is overexpressed. Finally, both quadruplex-forming oligonucleotides and many types of nanoparticles are easily modified to perform other functions, such as additional drug delivery, targeting to specific tissues, or even adding fluorescent or radioactive molecules for imaging and locating their biodistribution.

It is clear that many questions still remain in the field of quadruplex-forming oligonucleotides; however, the anti-cancer activity and other properties that they exhibit make them an attractive option for the treatment of cancer, justifying extensive future study and development. 


\section{REFERENCES}

Bates, P., Laber, D., Miller, D., Thomas, S., Trent, J. 2009. Discovery and development of the G-rich oligonucleotide AS1411 as a novel treatment for cancer. Experimental and Molecular Pathology 86:151-164.

Bryant, A., Cerfolio, R. 2007. Differences in epidemiology, histology, and survival between cigarette smokers and never-smokers who develop non-small cell lung cancer. Chest 132:185-192.

Cao, C., Wang, J., Bunjhoo, H., Xu, Y., Fang, H. 2011. Risk profile of bevacizumab in patients with non-small cell lung cancer: A meta-analysis of randomized controlled trials. Acta Oncologia Ahead of Print.

Chustecka, Zosia. "Addition of Sorafenib May Be Detrimental in Some Lung Cancer Patients.” Medscape Medical News. 25 April 2008.

Didenko, V. 2001. "DNA Probes Using Fluorescence Resonance Energy Transfer (FRET): Designs and Applications. Biotechniques 31:1106-1121.

“FDA Approval for Bevacizumab.” National Cancer Institute. November 18, 2011. $<$ http://www.cancer.gov/cancertopics/druginfo/fda-bevacizumab\#AnchorNSCLC> 
Gilmour, D., Fan, R. 2009. "Detecting transcriptionally engaged RNA polymerase in eukaryotic cells with permanganate genomic footprinting." Methods 48:368-374.

Gray, D., Ratliff, R., Vaughan, M. 1992. Circular dichroism spectroscopy of DNA. Methods in Enzymology 211:389-406.

Gubala, V., Betancourt, J., Rivera, J. 2004. Expanding the Hoogsteen edge of 2'deoxyguanosine: consequences for G-quadruplex formation. Organic Letters 6:4735-38.

Hilmi, C., Guyot, M., Pages, G. 2011. VEGF Spliced Variants: Possible Role of AntiAngiogenesis Therapy. Journal of Nucleic Acids 2012:1-7.

Huppert, J. 2008. Four-stranded nucleic acids: structure, function and targeting of Gquadruplexes. Chemical Society Reviews 37:1375-1384.

Jain, R., Duda, D., Clark, J., Loeffler, J. 2006. Lessons from phase III clinical trials on anti-VEGF therapy for cancer. Nature Clinical Practice Oncology 3:24-40.

Jiang, L., Schlesinger, F., Davis, C. 2011. Synthetic spike-in standards for RNA-seq experiments. Genome Research 21:1543-1551. 
Kurreck, T. 2003. Antisense technologies. European Journal of Biochemistry 270:16281644.

Loureiro, R., D'Amore, P. Transcriptional regulation of vascular endothelial growth factor in cancer. Cytokine \& Growth Factor Reviews 16:77-89.

“Lung Cancer - Non-Small Cell.” National Center for Biotechnology Information. $<$ http://www.ncbi.nlm.nih.gov/pubmedhealth/PMH0004462/>.

Mendel, D., Laird, A., Smolich, B., Blake, R., Liang, C., Hannah, A., Shaheen, R., Ellis, L., Weitman, S., Shawver, L., Cherrington, J. 2000. Development of SU5416, a selective small molecule inhibitor of VEGF receptor tyrosine kinase activity, as an anti-angiogenesis agent. Anti-Cancer Drug Design 15:29-41.

Molina, J., Adjei, A. Jett, J. 2006. Advances in Chemotherapy of Non-small Cell Lung Cancer. Chest 130:1211-1219.

Neidle, Stephan, and Shankar Balasubramanian. 2006. Quadruplex Nucleic Acids. Cambridge: The Royal Society of Chemistry.

“Non-Small Cell Lung Cancer Treatment." National Cancer Institute. June 23, 2011. $<$ http://cancer.gov/cancertopics/pdq/treatment/non-small-celllung/healthprofessional $>$. 
O’Byrne, K., Koukourakis, M., Giatromanolaki, A., Cox, G., Turley, H., Steward, W., Gatter, K., Harris, A. 2000. Vascular endothelial growth factor, platelet-derived endothelial cell growth factor and angiogenesis in non-small-cell lung cancer. British Journal of Cancer 82:1427-1432.

Olsson, A., Dimberg, A., Kreuger, J., Claesson-Welsh, L. 2006. VEGF receptor signaling - in control of vascular function. Nature Reviews 7:359-371.

Qin, Y., Hurley, L. 2008. Structures, folding patterns, and functions of intramolecular DNA G-quadruplexes found in eukaryotic promoter regions. Biochimie 90:11491171.

Rankin, S., Reszeka, A., Huppert, J., Zloh, M., Parkinson, G., Todd, A., Ladame, S., Balasubramanian, S., Neidle, S. 2005. Putative DNA Quaduplex Formation within the Human c-kit Oncogene. Journal of the American Chemical Society 127:10584-10589.

"Research Funding Statistics for Cancer Type." National Cancer Institute. $<\mathrm{http}$ //fundedresearch.cancer.gov/search/funded;jsessionid=9133F3620FCCA8F C626CEC0A0143B0E0?action=full\&fy=PUB2010\&type $=$ site $>$.

Robinson, C., Stringer, S. 2001. The splice variants of vascular endothelial growth factor (VEGF) and their receptors. Journal of Cell Science 114:853-856. 
Sedoris, K., Thomas, S., Clarkson, C., Muench, D., Islam, A., Singh, R., Miller, D. 2011. Genomic c-Myc Quadruplex DNA Selectively Kills Leukemia. Molecular Cancer Therapeutics Ahead of Print.

Travis, W., Travis, L., Devissa, S. 1995. Lung Cancer. Cancer 75:191-202.

Yuan, A., Yu, C., Luh, K., Chen, W., Lin, F., Kuo, S., Yang, P. 2000. Quantification of VEGF mRNA expression in non-small cell lung cancer using a real-time quantitative reverse transcription-PCR assay and a comparison with quantitative competitive reverse transcription-PCR. Laboratory Investigation 80:1671-1680.

Zhang, R., Wang, H. 2005. Antisense Technology. Cancer Gene Therapy 2005:35-49. 
Edward David Muench was born on October 24, 1987 in Edgewood, KY to Edward Joseph and Carol Jean Muench. He graduated from Covington Catholic High School in 2006 as a Governor's Scholar, and began his undergraduate career at the University of Louisville that fall. He completed the first of his co-ops at Blue Heaven Technologies in Louisville, KY, and the final two at the James Graham Brown Cancer Center in Louisville, KY. In July, 2010, he earned his Bachelor of Science degree with honors in Bioengineering.

During his time at the University of Louisville, he became a member of both the Sigma Chi Fraternity, serving as Interfraternity Council Representative, and the Biomedical Engineering Society, serving as secretary. Additionally, he was employed by the university tutoring service as a math and physics tutor. He also spent his time volunteering in the University of Louisville Hospital Emergency Room, with the Leadership Advantage program which benefits incoming engineering freshmen, at the Brown Cancer Center's Cancer Survivor Celebration, and at the American Cancer Society's Relay for Life.

Following graduation, David will return to his hometown, where he will be marrying the love of his life, Tricia. He will be a research assistant at the Cincinnati Children's Hospital Medical Center before beginning his M.D./Ph.D. training in pursuit of a career in medical oncology and cancer research. 